\title{
Clinical Experience and Recent Advances in the Development of Listeria-Based Tumor Immunotherapies
}

\section{OPEN ACCESS}

Edited by:

Stephanie E. B. McArdle, Nottingham Trent University, United Kingdom

Reviewed by:

Rodabe N. Amaria, University of Texas MD Anderson Cancer Center, United States

Graham Robert Leggatt, The University of Queensland, Australia

*Correspondence: Laurence M. Wood laurence.wood@ttuhsc.edu

Specialty section: This article was submitted to Cancer Immunity and Immunotherapy, a section of the journal Frontiers in Immunology

Received: 15 December 2020 Accepted: 26 February 2021

Published: 14 April 2021

Citation:

Oladejo M, Paterson Y and Wood LM (2021) Clinical Experience and Recent

Advances in the Development of

Listeria-Based Tumor

Immunotherapies.

Front. Immunol. 12:642316. doi: 10.3389/fimmu.2021.642316

\author{
Mariam Oladejo ${ }^{1}$, Yvonne Paterson ${ }^{2}$ and Laurence M. Wood ${ }^{1 *}$ \\ ${ }^{1}$ Immunotherapeutics and Biotechnology, Texas Tech University Health Sciences Center, Abilene, TX, United States, \\ ${ }^{2}$ Microbiology, Perelman School of Medicine, University of Pennsylvania, Philadelphia, PA, United States
}

The promise of tumor immunotherapy to significantly improve survival in patients who are refractory to long-standing therapies, such as chemotherapy and radiation, is now being realized. While immune checkpoint inhibitors that target PD-1 and CTLA-4 are leading the charge in clinical efficacy, there are a number of other promising tumor immunotherapies in advanced development such as Listeria-based vaccines. Due to its unique life cycle and ability to induce robust CTL responses, attenuated strains of Listeria monocytogenes $(\mathrm{Lm})$ have been utilized as vaccine vectors targeting both infectious disease and cancer. In fact, preclinical studies in a multitude of cancer types have found Listeria-based vaccines to be highly effective at activating anti-tumor immunity and eradicating tumors. Several clinical trials have now recently reported their results, demonstrating promising efficacy against some cancers, and unique challenges. Development of the $L m$-based immunotherapies continues with discovery of improved methods of attenuation, novel uses, and more effective combinatorial regimens. In this review, we provide a brief background of Listeria monocytogenes as a vaccine vector, discuss recent clinical experience with Listeriabased immunotherapies, and detail the advancements in development of improved Listeria-based vaccine platforms and in their utilization.

Keywords: Listeria monocytogenes, tumor immunotherapy, cancer vaccines, tumor antigens, vaccine vectors, clinical trials

\section{INTRODUCTION}

Tumor immunotherapy has gained rapid acceptance as an effective therapeutic strategy in the treatment of numerous malignancies. A multitude of methods to boost the anti-tumor immune response have been utilized including administration of purified immune system components to the use of microorganisms, such as attenuated bacteria and oncolytic viral particles, and have emerged as tools in the fight against cancer. While therapeutics that unleash the anti-tumor response by blocking inhibitory signaling pathways such as PD-1/PD-L1 have demonstrated the promise of tumor immunotherapy, immunotherapies that stimulate a specific anti-tumor response such as Sipuleucel-T, the first FDA-approved therapeutic cancer vaccine (1), have also provided a proof of 
concept for that approach. In fact, numerous cancer vaccines are currently in different stages of clinical trials with promising results (2).

Due to the challenge of overcoming tolerance within the tumor microenvironment, considerable effort has been directed towards stimulating the immune system to mount a robust response against these cells by targeting tumor-associated antigens (TAAs) and tumor-specific antigens (TSAs) (2). Necessary characteristics of an effective cancer vaccine are proficient antigen delivery, limited impact on normal healthy tissue, and the ability to elicit a robust anti-tumor immune response. In this regard, Listeria monocytogenes, a facultative gram-positive bacterium is an attractive platform for development of cancer vaccines due its ability to activate and deliver tumor antigens selectively to antigen-presenting cells, resulting in a robust antitumor cell-mediated immune response (3). It is these attributes of $L m$ that has driven significant development, in both academia and industry, of $L m$-based tumor immunotherapies with several candidates in various stages of clinical development (Table 1). Therefore, in this review, our focus will be on the use of $L m$ as a

TABLE 1 | Clinical Trials for $L C$-based Vaccines in Tumor Immunotherapy.

\begin{tabular}{|c|c|c|c|c|c|c|}
\hline Trial Status & $\begin{array}{l}\text { Lm-based } \\
\text { Vaccine }\end{array}$ & Targeted Antigen(s) & Disease & $\begin{array}{c}\text { Trial } \\
\text { Phase }\end{array}$ & Enrollment & $\begin{array}{c}\text { NCT } \\
\text { Number* }\end{array}$ \\
\hline \multirow[t]{8}{*}{ Active } & ADXS-NEO & $\begin{array}{l}\text { Multiple personalized } \\
\text { antigens }\end{array}$ & Multiple Cancers & Phase 1 & 5 & NCT03265080 \\
\hline & ADXS-HPV & HPV16 E7 & Cervical Cancer & Phase 3 & 450 & NCT02853604 \\
\hline & ADXS-PSA & PSA & Prostate Cancer & $\begin{array}{l}\text { Phase } \\
1 / 2\end{array}$ & 51 & NCT02325557 \\
\hline & ADXS-HPV & HPV16 E7 & Cervical, Head and Neck Cancer & $\begin{array}{c}\text { Phase } \\
1 / 2\end{array}$ & 66 & NCT02291055 \\
\hline & ADXS-HPV & HPV16 E7 & Oropharyngeal Cancer & Phase 2 & 15 & NCT02002182 \\
\hline & $\begin{array}{l}\text { ADXS-HOT } \\
\text { LUNG }\end{array}$ & $\begin{array}{l}\text { Multiple antigens (hotspot } \\
\text { mutations) }\end{array}$ & Non-Small-Cell Lung Cancer & $\begin{array}{c}\text { Phase } \\
1 / 2\end{array}$ & 74 & NCT03847519 \\
\hline & CRS-207 & Mesothelin & Pancreatic Cancer & Phase 2 & 63 & NCT03190265 \\
\hline & CRS-207 & Mesothelin & Pancreatic Cancer & Phase 2 & 70 & NCT03006302 \\
\hline \multirow{23}{*}{$\begin{array}{l}\text { Completed, } \\
\text { Withdrawn, } \\
\text { Terminated }\end{array}$} & ADXS31-164 & Her2 & HER2-Expressing Solid Tumors & $\begin{array}{l}\text { Phase } \\
1 / 2\end{array}$ & 12 & NCT02386501 \\
\hline & ADXS-HPV & HPV16 E7 & Anal, Rectal Cancer & Phase 2 & 51 & NCT02399813 \\
\hline & ADXS-HPV & HPV16 E7 & Anal Cancer & $\begin{array}{c}\text { Phase } \\
1 / 2\end{array}$ & 11 & NCT01671488 \\
\hline & ADXS-HPV & HPV16 E7 & Cervical Cancer & $\begin{array}{c}\text { Phase } \\
1 / 2\end{array}$ & 25 & NCT02164461 \\
\hline & ADXS-HPV & HPV16 E7 & Cervical Cancer & Phase 2 & 54 & NCT01266460 \\
\hline & CRS-207 & Mesothelin & Pancreatic Cancer & Phase 2 & 303 & NCT02004262 \\
\hline & ADU-623 & EGFRvIlI, NY-ESO-1 & $\begin{array}{l}\text { Astrocytic Tumors, } \\
\text { Glioblastoma Multiforme, } \\
\text { Anaplastic Astrocytoma, Brain Tumor }\end{array}$ & Phase 1 & 11 & NCT01967758 \\
\hline & $\begin{array}{l}\text { ANZ-100 } \\
\text { (CRS-100) }\end{array}$ & $\mathrm{N} / \mathrm{A}$ & Carcinoma and Liver Metastases & Phase 1 & 9 & NCT00327652 \\
\hline & $\begin{array}{l}\text { JNJ- } \\
64041809\end{array}$ & Multiple prostate antigens & Prostate Cancer & Phase 1 & 26 & NCT02625857 \\
\hline & ADXS-HPV & HPV16 E7 & Cervical Intraepithelial Neoplasia & Phase 2 & 81 & NCT01116245 \\
\hline & ADXS-HPV & HPV16 E7 & Oropharyngeal Carcinoma & Phase 1 & 2 & NCT01598792 \\
\hline & pLADD & $\begin{array}{l}\text { Multiple personalized } \\
\text { antigens }\end{array}$ & Colorectal Cancer & Phase 1 & 28 & NCT03189030 \\
\hline & CRS-207 & Mesothelin & Malignant Pleural Mesothelioma & Phase 1 & 60 & NCT01675765 \\
\hline & CRS-207 & Mesothelin & Malignant Pleural Mesothelioma & Phase 2 & 10 & NCT03175172 \\
\hline & CRS-207 & Mesothelin & Pancreatic Cancer & Phase 2 & 93 & NCT02243371 \\
\hline & CRS-207 & Mesothelin & Gastric, Gastroesophageal Junction, Esophageal Cancers & Phase 2 & 5 & NCT03122548 \\
\hline & CRS-207 & Mesothelin & $\begin{array}{l}\text { Malignant Epithelial Mesothelioma, Pancreatic, Ovarian, Non- } \\
\text { Small-Cell Lung Cancer }\end{array}$ & Phase 1 & 17 & NCT00585845 \\
\hline & CRS-207 & Mesothelin & Ovarian, Fallopian, Peritoneal Cancer & $\begin{array}{c}\text { Phase } \\
1 / 2\end{array}$ & 35 & NCT02575807 \\
\hline & CRS-207 & Mesothelin & Pancreatic Cancer & Phase 2 & 93 & NCT01417000 \\
\hline & $\begin{array}{l}\text { JNJ- } \\
64041757\end{array}$ & EGFRvIll, Mesothelin & Non-Small-Cell Lung Cancer & Phase 1 & 18 & NCT02592967 \\
\hline & $\begin{array}{l}\text { JNJ- } \\
64041757\end{array}$ & EGFRvIll, Mesothelin & Lung Cancer & $\begin{array}{c}\text { Phase } \\
1 / 2\end{array}$ & 12 & NCT03371381 \\
\hline & ADXS-HPV & HPV16 E7 & Non-Small-Cell Lung Cancer & Phase 2 & 124 & NCT02531854 \\
\hline & $\begin{array}{l}\text { JNJ- } \\
64041809\end{array}$ & Multiple prostate antigens & Prostate Cancer & Phase 2 & 0 & NCT02906605 \\
\hline
\end{tabular}

*Additional clinical trial information for each study available at https://clinicaltrials.gov/ct2/home. 
tumor immunotherapy vaccine platform including a review of recent advancements in construction of improved $L m$-based tumor immunotherapies, efficacy of $L m$-based tumor immunotherapies in combination with other anti-cancer treatment modalities, and the current clinical experience with $L m$-based tumor immunotherapies.

\section{DEVELOPMENT OF INACTIVATED AND ATTENUATED BACTERIA AS TUMOR IMMUNOTHERAPEUTICS}

Evidence that bacteria can harness the immune system to elicit therapeutic efficacy against malignancies dates to the early twentieth century. While William Coley laid a solid foundation for modern bacterial immunotherapy, there are other notable scientists whose work predated even his discoveries. In 1851, Belgian surgeon Didot pioneered the use of a syphilis vaccination to treat inoperable cancer (4). In 1868, Busch, a German scientist, reported the efficacy of erysipelas infection in treatment of sarcoma in a crudely performed clinical study (5-7). Two decades later in 1882, Friedrich Fehleisen discovered Streptococcus pyogenes to be the causative agent of erysipelas and noticed that infection with this bacterium caused transplanted tumors to melt away $(5,6,8)$. It was armed with these observations and the serendipitous recovery of a German immigrant with an inoperable neck sarcoma, that William Coley embarked on the challenging but foundational work of tumor immunology (9). His belief that a component or "factor" from the microbes, rather than the whole live microorganism, is responsible for the oncolytic activity he observed led to the creation of his Coley toxins, a mixture of heat killed Streptococcus pyogenes and Serratia mersacems (9). In fact, this novel therapy was reported to result in partial or complete remissions in many difficult-to-treat patients $(10,11)$. However, given our limited understanding of immunology at the time, the mechanisms that would explain the efficacy of this strategy were unclear, thereby limiting the continued development of Coley's toxins. Eventually, the advent of radiotherapy and chemotherapy that could be more easily standardized relegated this novel therapeutic strategy, comprised of killed or inactivated bacteria, to the background. As our understanding of the immune system has matured, there has been renewed interest in the use of attenuated, inactivated, and killed bacteria to stimulate anti-tumor immunity.

\section{USE OF LISTERIA MONOCYTOGENES AS A TUMOR IMMUNOTHERAPEUTIC TO ELICIT TUMOR SPECIFIC IMMUNITY}

Unlike the approach taken by Coley, whose toxins non-specifically resulted in an anti-tumor response, current approaches focus on achieving complete and durable antitumor immunity through induction of tumor-specific immune responses. To elicit this tumor-specific immunity, the strategy employed by most tumor immunotherapies is to activate cytotoxic T lymphocytes (CTLs) that recognize peptides from tumor-specific and/or tumorassociated antigens presented on MHC Class I and target tumor cells for lysis (12). In fact, CTL-mediated tumor cell destruction occurs commonly in a process called immunosurveillance, in which the immune system recognizes and eliminates malignant cells prior to clinical detection (13). Unfortunately, some malignant cells can escape immune-mediated destruction and develop into a clinically relevant tumor. The tumors that have escaped immunosurveillance have been able to achieve this, in part, by fostering the development of an immunosuppressive microenvironment that impedes the function and survival of responding lymphocytes, including cytotoxic $\mathrm{T}$ lymphocytes. In order to overcome this immunosuppressive microenvironment, various strategies have been employed to enhance the activation and mobilization of CTLs in the tumor environment such as the use of oncolytic viruses, dendritic cell vaccines, adoptive cell therapy, and microbial vectors for the targeting of TAAs (14-16). Each of these strategies has their own advantages and disadvantages. Aside from the important consideration of toxicity in the use of oncolytic viruses, off target viral replication and development of neutralizing antibodies may prevent recurrent use of this strategy. Bacterial vectors such as Streptococcus pyogenes, Clostridium novyi, Salmonella enterica, and Listeria monocytogenes (17-20), are all vectors that have been used in cancer immunotherapy and do not pose this significant drawback of preexisting neutralizing immunity. However, what differentiates Listeria monocytogenes from its vaccine peers and makes it a superior vector for delivery of cancer antigens is its unique life cycle. $L m$ is readily taken up by macrophages in the course of an infection and, once within a phagocytic compartment, expresses and secretes a cytolysin, Listeriolysin O (LLO) (21). LLO, along with bacterial phospholipases, disrupts the integrity of the phagosome and allows $L m$ to escape into the cytosol and elude destruction in the phagolysosome (22). Once in the cytosol, $\mathrm{Lm}$ proliferates and secretes additional virulence factors that propel it within the cell and into adjacent cells in order to propagate the infection (23). It is this life cycle that makes $\mathrm{Lm}$ an ideal candidate to deliver antigen to both the MHC I and II pathways, activating $\mathrm{CD}^{+} \mathrm{T}$ cells and, most importantly for tumor-lytic responses, cytotoxic $\mathrm{CD}^{+} \mathrm{T}$ cells (24). In fact, infection with $\mathrm{Lm}$ elicits a robust and long-lasting immunological memory response that provides protection against infection upon future exposure to the pathogen (25-27).

In addition to its unique life cycle, $L m$ affords a number of advantages as a vector for tumor immunotherapy. Previous studies found that antigens encoded by $\mathrm{Lm}$ constructs are more efficiently delivered to the protein processing and presentation machinery than those encoded by other bacterial vectors (28). Further, $L m$ vectors have the ability to break immunologic tolerance, via reduction of immunosuppressive myeloid-derived suppressor cells (MDSCs) and regulatory T cells (Tregs) within the tumor microenvironment. Finally, in addition to selective uptake in the spleen and liver of infected subjects, $L m$ displays a specific tropism for primary and metastatic tumors $(29,30)$. These attractive features, including the ease of manipulation and attenuation of this organism, have been harnessed by various 
groups in development of attenuated strains of $L m$, expressing a wide variety of tumor antigens. We have extensively discussed the various ways by which these attenuated strains are generated, and the antigens that have been delivered by this therapeutic platform
(31). Described below, are the more recent $\mathrm{Lm}$ construction trends as well as the use of these various constructs in combination therapy depicted in Figure $\mathbf{1}$ that are currently being tested in various preclinical and clinical trials (Figure 1).

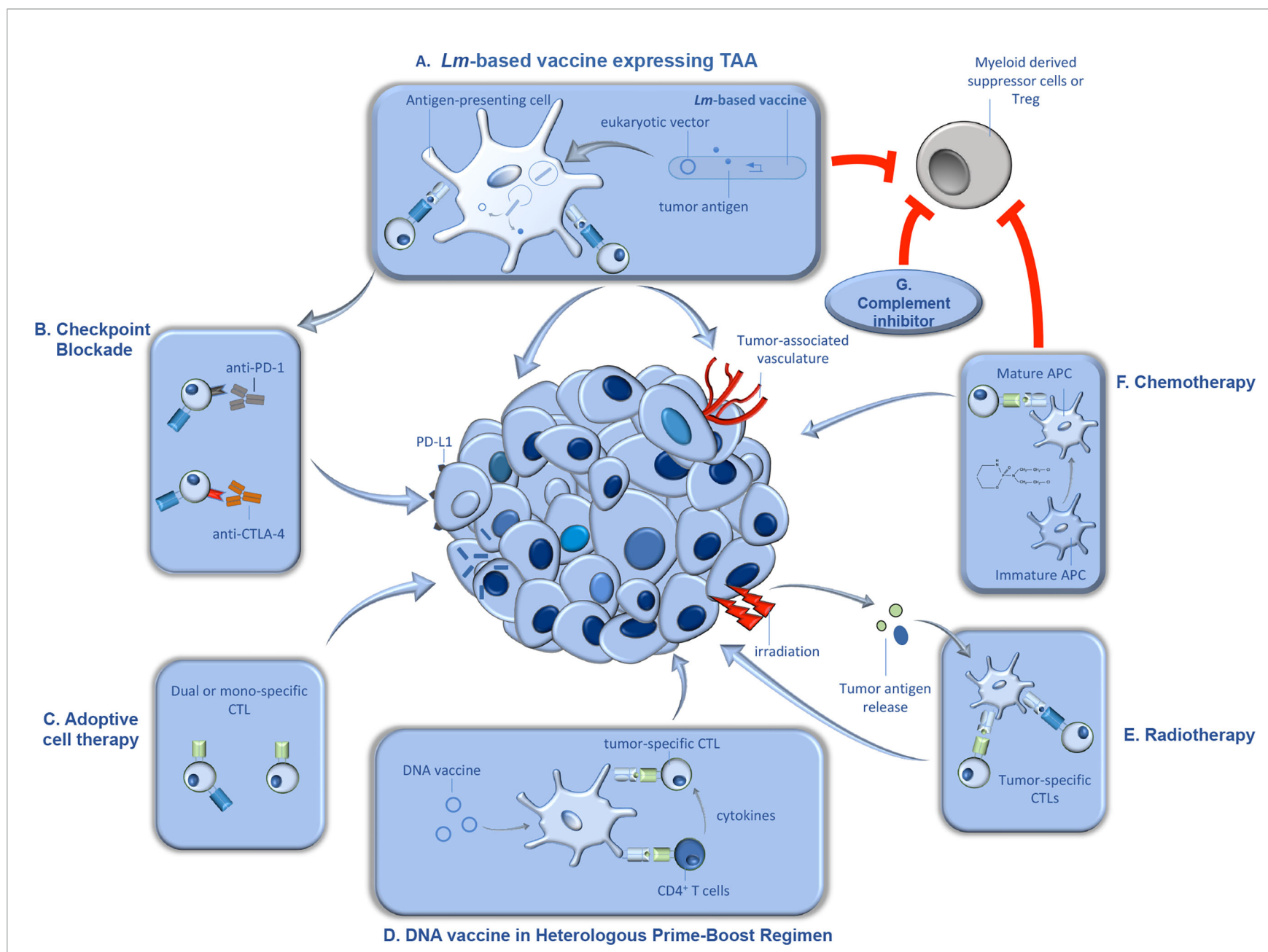

FIGURE 1 | Synergistic therapeutic approaches with $L m$-based vaccines in tumor immunotherapy. $L m$-based vaccines have found therapeutic success in preclinical models of cancer for decades, and recent studies demonstrated significant promise for this type of active tumor immunotherapy in clinical trials. Further, recent studies suggest that the anti-tumor efficacy of $L m$-based vaccines can be significantly improved when utilized in combination with synergistic anti-cancer therapeutics. In this figure, we detail some of the anti-cancer therapeutics that demonstrated efficacy in combination $L m$-based vaccines along with their proposed mechanism(s) of action. (A) Upon administration, attenuated $L m$ vaccines infect antigen presenting cells in secondary lymphoid organs and gain entry to the cytosol, wherein they produce and secrete tumor antigen and/or release eukaryotic expression vectors encoding for tumor antigen. Once delivered by $L m$, the tumor antigens go through processing and presentation to naïve tumor-specific CD4 ${ }^{+}$and $\mathrm{CD} 8^{+}$cytotoxic T lymphocytes (CTLs). Tumor-specific CTLs, activated through the action of $L m$-based vaccines, migrate to the tumor microenvironment (TME) and lyse tumor cells and/or cells associated with the tumor vasculature. Importantly, Lm-based vaccines can also reduce immunosuppression within the TME by reducing tumor-associated MDSCs and Tregs. (B) Upon continuous activation, tumorspecific CTLs can become exhausted, characterized by the upregulation of inhibitory molecules such as programmed cell death protein 1 (PD-1) and cytotoxic T-lymphocyte-associated protein 4 (CTLA-4). Antibody-mediated blockade of inhibitory molecules results in enhanced T cell function and synergy with $L m$-based vaccine anti-tumor responses. (C) Dual or mono-specific adoptively transferred CTLs targeting both tumor antigens and/or $L m$-derived antigens work in concert with $L m$-based vaccines, delivered intratumorally, through increasing the breadth of the anti-tumor $T$ cell response. (D) $L m$-based vaccines have also been found to be effective in heterologous prime-boost approaches. DNA-based tumor vaccines, used as either prime or boost in a heterologous prime-boost vaccination schedule with $L m$-based vaccines, induce robust expansion of Th1 helper T cells that produce cytokines in support of responses by tumor-specific CTLs. (E) Further, radiation can synergize with $L m$-based vaccines, in part, by causing direct tumor death, resulting in release of tumor antigens which are processed and presented by APCs to tumor-specific T cells. (F) Chemotherapeutic agents such as cyclophosphamide can also synergize with $L m$-based vaccines, in part, by facilitating maturation of APCs. However, cyclophosphamide can also reduce immunosuppressive cell types, Tregs and MDSCs, in the TME allowing for improved anti-tumor efficacy by the anti-tumor responses induced by $L m$-based vaccines. (G) Similarly, inhibition of complement signaling can synergize with $L m$-based vaccines, in part, through limiting recruitment of MDSCs and Tregs to the TME. 


\section{CLINICAL EXPERIENCE WITH LM-BASED VACCINES FOR CANCER}

With the clinical experience of $L m$-based vaccines in oncology going well beyond a decade, the promise and further challenges are now being realized regarding their place in the future oncologists toolkit. Below, we summarize clinical studies that have recently published their findings for an array of cancers. Importantly, these studies only scratch the surface of clinical studies regarding the efficacy of $L m$-based vaccines for cancer that are currently underway or not yet peer-reviewed and published as summarized in Table $\mathbf{1}$.

\section{HPV-Associated Cancers}

As the development of $L m$-based vaccine vectors matured in the late 1990's, the focus of constructing novel vectors progressed from those that targeted model tumor antigens, in order to better understand the platform, to the incorporation of clinically relevant tumor antigens. One of the first $L m$-based vaccines to incorporate a clinically relevant tumor antigen targeted Human papillomavirus (HPV)-associated cancers (3). While most individuals clear infections with HPV, in certain individuals that are unable to clear the infection, chronic infection with high-risk strains of HPV, such as HPV 16 and 18, can increase their risk of developing cancer (32). Infection with high-risk HPV is particularly problematic in women as it can result in carcinoma of the cervix (33). However, high-risk strains of HPV are also associated with head and neck cancers among others (34). In HPV-associated cancers, constitutive expression of the HPV oncoproteins, E6 and E7, is required to maintain the malignant phenotype (35). Therefore, this necessary expression by the tumor cells, and the lack of central tolerance to these viral antigens, make these oncoproteins particularly attractive targets for $L m$-based tumor immunotherapy.

In the initial preclinical study documenting the development of $L m$-based vaccines targeting $\mathrm{HPV}$-associated cancers, there were two vaccines constructed, Lm-E7 and Lm-LLO-E7 (3). While Lm-E7 expressed and secreted HPV 16 E7, generated E7-specific CTLs, and demonstrated anti-tumor efficacy, this was insufficient to cure a majority of mice with established tumors expressing E7. However, administration with an $\mathrm{Lm}$ construct that expressed and secreted HPV 16 E7 genetically fused to a truncated non-hemolytic form of Listeriolysin O (tLLO) did result in complete eradication of E7-expressing tumors in all experimental mice. The adjuvant-like properties of tLLO have since been elucidated and the vast majority of $L m$-based vaccines in clinical and preclinical testing genetically fuse the tumor antigen to either tLLO or a truncated form of another $\mathrm{Lm}$ derived protein with adjuvant-like properties, ActA (36-39).

The first clinical use of Lm -LLO-E7 (also known as ADXSHPV and as ADXS11-001), or any Lm-based therapy in cancer patients, was a Phase I clinical trial in women with cervical cancer that had failed previous front-line therapy including surgery, chemotherapy, and radiation (40). The patients were enrolled into three groups of 5 with each group receiving two intravenous doses ranging from $10^{9}$ colony-forming units (CFU) of Lm-LLO-E7 to $10^{10} \mathrm{CFU}$ given 22 days apart. As would be expected with intravenous (i.v.) administration of $L m$, all of the patients experienced adverse events (AE) including fever, chills, nausea, and vomiting with $40 \%$ experiencing severe grade 3 AEs but none of the patients experienced a life-threatening grade 4 adverse event (AE) due to the treatment. Dose-limiting toxicities occurred in the group receiving the highest dose with one patient requiring antibiotics to mitigate a fever. In terms of efficacy, nearly half of the patients were reported to have stable disease with one receiving a possible partial response and the median overall survival was 347 days. These results warranted further investigation as they demonstrated that $L m$-based vaccines have a safety profile that is likely more favorable than salvage chemotherapy and efficacy that may improve upon current treatment modalities.

Following the promising safety profile of Lm-LLO-E7 observed in the Phase I clinical trial, two Phase II trials were commenced to further characterize its safety and efficacy in patients with recurrent cervical cancer. In the first trial, 109 patients were separated into two groups with one receiving only $10^{9} \mathrm{CFU} L m$-LLO-E7 and the second group receiving Lm-LLOE7 along with cisplatin (41). While the Lm-LLO-E7 group received three i.v. administrations each separated by roughly one month, the Lm-LLO-E7 + cisplatin group received their initial dose of $L m$-LLO-E7 and then waited 4 weeks to receive their 5 weekly doses of cisplatin and finally the second dose of Lm-LLO-E7. The rationale for this staggered administration of Lm-LLO-E7 and cisplatin was to promote treatment synergy while limiting any possible suppressive effect of cisplatin on the immune response to Lm-LLO-E7. However, the addition of cisplatin to Lm-LLO-E7 did not have a significant impact on efficacy. The overall response rates (ORR) were comparable between the groups with an ORR of $17.1 \%$ for the $\mathrm{Lm}$-LLO-E7 group and $14.7 \%$ for the $L m$-LLO-E7 + cisplatin group. Similarly, median overall survival (OS) were not statistically significant with 8.28 and 8.78 months in the Lm-LLO-E7 and Lm-LLO-E7+ cisplatin groups, respectively. In terms of safety, the difference between these two arms was more pronounced with more AEs observed in the Lm-LLO-E7+cisplatin group (429) than the LmLLO-E7 alone group (275) but most of these AEs were mild to moderate in severity (80.4\%). These results informed the second Phase II trial for cervical cancer funded by the NCI, wherein, there was only a single arm receiving three doses of $L m$-LLO-E7 only in the first stage, with some patients receiving an additional three doses in a second stage (42). Treatment-related AE types and frequencies were similar to previous trials with nearly all patients experiencing at least one and $38 \%$ experiencing a grade $3 \mathrm{AE}$. In addition, $4 \%$ of patients (2) experienced a grade $4 \mathrm{AE}$ that consisted of sepsis and cytokine release syndrome. In terms of efficacy, the median OS was 6.1 months and the 12-month OS rate was $38 \%$ which met the study goal of $35 \%$, a dramatic improvement on the historical 12 -month OS of $21 \%$ in a similar patient population. This trial along with other ADXS-HPV trials were placed on temporary hold when evidence of listeriosis was observed in a patient 31 months after the last administration. This was believed to be due to persistence 
associated with biofilm formation on prosthetic material at recent fracture sites in the patient (43). These Phase II trials demonstrated that $\mathrm{Lm}$-LLO-E7 is tolerated relatively well in comparison to other treatment modalities and has significant promise to extend the lifespan of a poorly treated patient population.

The results from the Phase I and Phase II clinical trials of $\mathrm{Lm}$ LLO-E7 in cervical cancer resulted in the commencement of a Phase III clinical trial, AIM2CERV (NCT02853604). While AIM2CERV is still ongoing and results have not yet been published, the recruitment of new patients has been halted by its sponsor, Advaxis Inc., in order to allocate resources to the development of other promising upcoming vaccine platforms. However, clinical trials are still ongoing with Lm-LLO-E7 to evaluate the efficacy of Lm-LLO-E7 in other HPV-associated cancers, but the halt of AIM2CERV suggests it may take longer than previously anticipated before possible FDA approval and wide availability of $\mathrm{Lm}$-LLO-E7.

In addition to cervical cancer, a recent study reported promising results with $\mathrm{Lm}$-LLO-E7 in another HPV-associated cancer, anal cancer (44). In this Phase 1/2 clinical trial, patients with non-metastatic anal cancer were administered $\mathrm{Lm}$-LLO-E7 once before and three times after receiving chemoradiation consisting of mitomycin C, 5-fluorouracil, and daily imageguided radiation therapy. While most patients experienced only low-grade AEs due to the $L m$-based vaccine, two patients did experience grade 3 chills/rigor but all AEs were managed and resolved within $24 \mathrm{hrs}$. The clinical response was also highly promising as all 9 patients experienced complete responses by trial end (42 months) with only one patient progressing to metastatic disease six months post-trial. This study suggests Lm-LLO-E7 may have a promising future as part of a frontline therapeutic regimen in the treatment of HPV-associated cancers beyond cervical cancer.

\section{Pancreatic Cancer}

Pancreatic ductal cell carcinoma (PDAC) is one of the most challenging malignancies to treat with the most effective current treatments being surgery and chemotherapy (45). Unfortunately, while the advancement of immunotherapy in oncology, particularly immune checkpoint inhibition (ICI), has brought about significant survival benefits to many cancer patients, PDAC patients have not been afforded the same benefit (46). PDAC expresses TAAs such as mesothelin but is poorly infiltrated by $\mathrm{T}$ cells and is considered an immunologically "cold" tumor (47-49). As $L m$-based vaccines have been found to modulate the tumor microenvironment (TME) and make it more amenable to anti-tumor immune responses (50), significant effort has been expended to determine the promise of this vaccine platform in the treatment of PDAC. To target mesothelin-expressing PDAC, an $L m$-based vaccine was constructed that expressed and secreted human mesothelin genetically fused to the first 100 residues of ActA, ActAN100, in a highly attenuated strain of $L m$, LADD (51). This mesothelintargeting $L m$-based vaccine ( $L m$-Mesothelin, CRS-207) was initially brought into the clinic in a Phase I trial with several cancer types that all express mesothelin, including PDAC (52).
Study subjects received at least one i.v. administration of CRS207 at a particular dose ranging from $10^{8}$ to $10^{10}$ CFUs. The vaccine was generally well tolerated with the majority of patients experiencing grade 2 or less AEs. However, a number of patients experienced grade 3 AEs and at least 2 grade 4 AEs at the highest doses. Therefore, the maximum tolerated dose was determined to be $10^{9}$ CFUs. While the study was not powered to assess efficacy, $37 \%$ of patients survived beyond 15 months. Interestingly, the patients with longer survival had a more robust $\mathrm{T}$ cell response to the vaccine.

Due to the promising data from the Phase I clinical trial, three separate Phase II clinical trials were commenced to evaluate the efficacy and safety of CRS-207 in PDAC. The first Phase IIa trial with 90 patients assessed the efficacy of cyclophosphamide (Cy), a chemotherapeutic drug that limits peripheral tolerance, in combination with GVAX, a tumor vaccine consisting of irradiated allogeneic PDAC cells lines secreting GM-CSF (Arm B) or a heterologous prime boost regimen with Cy/GVAX with CRS-207 (Arm A) (53). The heterologous prime boost regimen, Cy/GVAX+CRS-207, was superior to the Cy/GVAX only treatment regimen, in terms of median OS (6.1 vs. 3.9 months) and in terms of 12 -month survival (24\% vs. $12 \%)$. In fact, mesothelin-specific $\mathrm{CD}^{+} \mathrm{T}$ cell responses were observed earlier with Cy/GVAX+CRS-207 than Cy/GVAX only suggesting that the expected synergy of the prime boost approach was realized in terms of both efficacy and anti-tumor immune response. The most common AEs were associated with injection site reactions but a majority of patients also experienced fever, chills, and gastrointestinal symptoms. The most prevalent severe AE (grade 3-4) was lymphopenia that was observed in $44 \%$ of patients. However, one patient did develop systemic listeriosis 12 days post administration that resolved after receiving i.v. penicillin (54). The promising results demonstrate the ability of an $L m$-based vaccine to synergize with another vaccine strategy, in this case Cy/GVAX, in a heterologous prime boost regimen, thereby, providing strong rationale for the continued exploration of the ability of immunotherapy to increase survival in PDAC.

Unlike the first Phase IIa clinical trial with CRS-207, the second trial (ECLIPSE study) included groups to assess the therapeutic efficacy of CRS-207 by itself in comparison to standard-of-care chemotherapy and a CRS-207+Cy/GVAX combination treatment regimen (55). The primary group in this study was patients given each of these interventions as a third+ line therapy. In this population, the median OS of chemotherapy alone was 4.6 months vs. 3.7 months with the Cy/GVAX+CRS-207 regimen. Interestingly, the CRS-207 alone treatment group had a median OS of 5.4 months suggesting some promise as a therapy in PDAC but these differences in median OS were not significant between any of the treatment groups. In a smaller group of patients when each of these treatment regimens were given as a second line therapy, there was no significant difference in median OS but the chemotherapy treatment arm had the highest median OS. The lower median OS in this trial compared to the first Phase II trial with CRS-207 was likely due to the patients in this trial having more advanced disease. As with the previous CRS-207 studies, treatment related severe AEs were 
minimal with the majority of AEs being low-grade. This trial again demonstrates the challenges of demonstrating efficacy in PDAC and suggested that a different approach may be required to demonstrate the promise of $L m$-based vaccines in this disease.

As the previous Phase II studies with CRS-207 suggested, a new therapeutic approach would be required to demonstrate that it is capable of improving PDAC patient survival. As ICI therapy has been found to synergize with $L m$-based vaccine approaches preclinically, a third Phase II trial with CRS-207 was conducted in PDAC patients that included anti-hPD-1 blockading antibody, nivolumab, along with Cy/GVAX+CRS-207 (Arm A) in comparison to Cy/GVAX+CRS-207 alone (Arm B) (56). The median OS for each arm was similar at 5.9 and 6.1 months for Arm A and Arm B, respectively. While the addition of nivolumab did not appear to improve median OS in patients receiving Cy/GVAX+CRS-207, some other parameters such as disease control rate and 12- and 18-month survival were improved beyond Cy/GVAX+CRS-207 alone. Interestingly, in the patients receiving nivolumab, long-term survival was correlated with increased $\mathrm{CD}^{+} \mathrm{T}$ cell infiltration into tumors and a reduction in likely immunosuppressive tumor-associated myeloid cells. However, nivolumab treatment did result in more AEs in patients but these were as expected in ICI therapy with $2.2 \%$ of patients, all in Arm A, discontinuing treatment due to treatment-related AEs. This study again highlights the enormous challenges of improving survival in PDAC patients. While the Phase II clinical studies with CRS-207 in PDAC did not meet their primary efficacy endpoints, they have advanced our knowledge and experience with $L m$-based vaccines in a particularly difficult-to-treat patient population that will hopefully inspire additional studies with improved treatment regimens.

\section{Malignant Pleural Mesothelioma}

Malignant pleural mesothelioma is a rare disease caused by exposure to asbestos with a high mortality rate (57). The previous first line therapy for MPM was pemetrexed with cisplatin that afforded patients a median OS of 14.1 months, until the recent approval of nivolumab and ipilumimab that raised median OS to 18.1 months in the Checkmate-743 Phase III trial $(58,59)$. The clinical success of ICI therapy in MPM suggests that it is a disease that is receptive to immunotherapies such as $L m$-based vaccines. As CRS-207 targets mesothelin, a tumor antigen highly expressed in MPM, a Phase I clinical trial was conducted to determine safety and tolerability of CRS-207 in combination with pemetrexed and cisplatin. In contrast to its efficacy on PDAC, 89\% of patients receiving CRS-207 in combination with pemetrexed and cisplatin experienced tumor reduction (60). While the median OS was comparable to chemotherapy at 14.7 months, the OS rate at 1 year of $64.9 \%$ was more comparable to ICI-treated MPM patients. As a correlate to this promising efficacy, percentages of functional $\mathrm{CD}^{+} \mathrm{T}$ cells increased and immunosuppressive M2 macrophages decreased in tumors over the course of treatment. AEs were similar to previous trials with CRS-207 and there was no additive effect with the addition of chemotherapy. While only a Phase I study with less than 40 patients, the promising results provide a strong rationale for the continued assessment of CRS-207 in MPM and possibly in combination with the recently FDA-approved ICI therapeutics to determine any synergistic potential.

\section{Non-Small Cell Lung Cancer}

Lung cancer is responsible for more deaths worldwide than any other form of cancer with non-small cell lung cancer (NSCLC) accounting for the vast majority (61-63). Effective therapeutic options are lacking for NSCLC as even ICI therapy provides relatively limited benefits compared to other cancers, with a 5-year survival of $15-25 \%(64,65)$. Due to the previously reported ability of $L m$-based immunotherapies to reduce immunosuppression in the TME and activate tumor-specific immunity, a Phase Ib/2 trial was commenced to determine the safety and effectiveness of $\mathrm{Lm}$ based vaccination alone or $L m$-based vaccination in combination with ICI in NSCLC (66). The bivalent $L m$-based vaccine utilized in these trials, JNJ-75, expresses NSCLC-associated antigens, both EGFRvIII and mesothelin, on the LADD vector platform, and was previously developed by Aduro Biotech as ADU-214 (31). As a monotherapy, 18 patients received JNJ-75 administered twice i.v. in either $10^{8}$ or $10^{9} \mathrm{CFUs/dose.} \mathrm{Each} \mathrm{dosage} \mathrm{of} \mathrm{JNJ-75} \mathrm{was} \mathrm{well}$ tolerated with no dose-limiting toxicity, and the expected pyrexia and chills with duration no longer than $24 \mathrm{hrs}$. Similar safety data was seen in the combination study where all 12 patients received $10^{9}$ CFUs of JNJ-75 along with $240 \mathrm{mg}$ nivolumab. In total, only 6 treatment-related serious AEs (grade 3 or higher) were observed and bacterial shedding was not found in blood, fecal, or urine samples at $4 \mathrm{hrs}, 2$ days, and 4 days-post administration. In terms of clinical response, $44 \%$ of patients had stable disease as the best overall response while one patient had a partial response in a target lesion identified at the beginning of the trial but still had overall disease progression. In the combination trial, the best overall response was stable disease in 4 patients but the trial was stopped early and efficacy data was limited. In the monotherapy study, levels of serum proinflammatory cytokines were elevated and activation of T cells and NK cells was increased at $24 \mathrm{hrs}$ post administration with cytokine levels returning to baseline at $48 \mathrm{hrs}$. Further, the magnitude of mesothelin-specific $\mathrm{T}$ cells responses was limited compared to recall responses to tetanus toxoid and influenza. While stable disease was observed in some patients and there was clear evidence of inflammatory responses, the overall lack of significant clinical benefit to patients suggests that JNJ-75 alone or in combination with nivolumab will not proceed to further with clinical development.

\section{Osteosarcoma}

Osteosarcoma is a highly aggressive form of cancer that overwhelmingly affects children and primarily manifests in the long bones prior to metastasizing to vital organs (67). The standard of care currently consists of resection of the lesion or amputations of the affected limb and chemotherapy (68). However, many patients experience recurrence of the disease due to the seeding of micrometastases prior to primary tumor resection (69). Much like in breast cancer, a proportion of pediatric osteosarcoma patients have lesions that highly express the EGFR family receptor, HER2/neu, that correlates with poor prognosis (70). Importantly, a Phase Ib clinical trial 
has recently completed utilizing a human Her2/neu-targeting Lm-based vaccine, ADXS31-164, for adult patients with Her2/ neu-expressing tumors (NCT02386501). This vaccine has now been licensed for development in the pediatric osteosarcoma setting by OS Therapies in conjunction with the NCI Children's Oncology Group. While human clinical studies have not yet begun targeting human osteosarcoma with $\mathrm{Lm}$-based immunotherapy, the promise of this therapeutic approach in humans may be predicted by the recently completed and published results from clinical trials in canines for osteosarcoma utilizing ADXS31-164. Canine osteosarcoma is highly aggressive much like the human disease in terms of prognosis and treatment (71). In fact, a significant proportion of canine osteosarcomas overexpress the tumor antigen Her2/ neu much like their human counterparts $(70,72)$. Since human Her2 and canine Her2 have $>90 \%$ homology and the $L m$-based vaccine targeting human Her2/neu, ADXS31-164, had already been developed and successfully tested in mice, a small Phase I clinical trial consisting of 18 client-owned dogs was performed to determine the safety, tolerability, and efficacy of the Lm-based vaccine targeting Her2/neu, ADXS31-164, in canine osteosarcoma $(73,74)$. For this trial, dogs were treated with three doses of ADXS31-164 once every three weeks following standard of care amputation and carboplatin adjuvant chemotherapy. AEs were generally low-grade, transient, and independent of ADXS31-164 dosage. Strikingly, the 1, 2, and 3-year survival rates for ADXS31-164 treated dogs were $77.8 \%, 67 \%$, and $56 \%$, respectively. This is in stark contrast to dogs in a historical control group treated with standard of care amputation and adjuvant chemotherapy with 1,2 , and 3-year survival rates of $55 \%, 28 \%$, and $22 \%$, respectively. In addition to increased survival, Her2-specific $\mathrm{T}$ cell responses were also observed in $83 \%$ of the dogs in the study. Based on the promising results of this study, ADXS31-164 was granted conditional approval by the USDA and the study was continued and expanded. In fact, a subsequent study of ADXS31-164 consisting of 50 dogs with osteosarcoma was recently reported. The study protocol mimicked the early Phase I trial and the observed AEs generally confirmed the low-grade and transient nature of those reported in the first study (75). However, 4 dogs did develop listeriosis, a finding not observed in the initial trial. The severity of the listeriosis varied between the animals with some requiring antibiotic treatment. One animal presented with an extrapleural mass that cultured positive for ADXS31-164 three weeks after receiving the final vaccine dose, and required surgery to remove the chest abscess (76). Unfortunately, the study was unable to report efficacy data due to $30 \%$ of the dogs discontinuing treatment, an inability to complete follow-up evaluations, and concurrent or subsequent therapies after ADXS31-164 treatment that complicated analysis.

\section{Future of Listeria in the Clinic}

Clinical trials continue for a number of $L m$-based vaccines (Table 1). However, with the discontinuation of the AIM2CERV Phase III trial, it may take longer than previously anticipated before an $L m$-based vaccine is widely available for use in oncology. While the safety profile of $L m$-based vaccines has been very tolerable in comparison to chemotherapy and other forms of immunotherapy, the recent rare reports of listeriosis after final dosing in both humans and dogs may be a challenge in a small number of patients going forward $(43,76)$. As current trials already incorporate a regimen of antibiotics subsequent to dosing with $L m$-based vaccines, this regimen may need to be modified and extended in order to mitigate the possible risk of listeriosis. Further, development of even more attenuated vaccine strains described below may help further mitigate this risk and, along with the combination of synergistic therapies, may spur greater clinical success for future $\mathrm{Lm}$-based vaccines.

\section{NEW TRENDS IN LM VACCINE CONSTRUCTS}

For more than two decades, Listeria monocytogenes has proven itself to be a highly promising vector for tumor immunotherapy in numerous preclinical studies (31). While the currently available platforms have demonstrated promising performance in clinical trials, none has yet been approved for clinical use by the FDA, thus there is continued interest in engineering newer, safer and more effective Listeria monocytogenes-based vaccine platforms (77-79). In addition, while numerous clinical studies have confirmed an excellent safety profile for $L m$-based vaccines in healthy individuals and oncology patients, there have been some concerns regarding the ability of current clinical strains to persist in patients $(40,43,52,66,76,80)$. Described below are the recent advances at overcoming these challenges and improving the safety and efficacy of $L m$-based immunotherapies

\section{Enhanced Attenuation Methods}

While infection with the virulent wild-type strain of Listeria monocytogenes can lead to the formation of robust memory $\mathrm{T}$ cell responses, several studies have found that attenuated strains of $\mathrm{Lm}$ actually result in improved immune memory and protective responses $(81,82)$. The methods utilized to attenuate $\mathrm{Lm}$ primarily revolve around deletion of non-essential virulence genes that allow for sufficient infectivity and antigen production but limit the potential for severe infection, a necessary concern when the patient population for tumor immunotherapy may already suffer from immune deficiency (83). In fact, the $L m$-based vaccines currently or previously in clinical trials have all contained some form of deletion of virulence genes such as actA or prfA (31). While the safety of these attenuated $\mathrm{Lm}$ platforms has been demonstrated in multiple trials, further modifications that enhance attenuation and improve therapeutic efficacy of $L m$-based vaccines continue.

One such advancement is the development of suicidal strains that are programmed to lose viability once the antigenic cargo has been delivered to an antigen-presenting cell (84). The need for these suicidal vaccines stems from instances where, despite attenuation, persistence of $L m$ vaccine constructs has been observed following the administration of live $\mathrm{Lm}$ vaccines (43, 85). Early work in the development of a Listeria suicide strain utilized an $L m$ construct that produced a phage lysin under the 
control of the ActA promoter so that the suicide switch would be engaged once $\mathrm{Lm}$ gained entry to the cytosol of an APC and delivered its cargo (84). A similar approach by Souders et al, that we discuss further in 1.4.4, found a dramatic reduction in death of infected APCs by a suicidal strain of $\mathrm{Lm}$ while still retaining the ability to successfully deliver a eukaryotic plasmid for expression of tumor antigen (79). Recently, this concept of an Lm-based suicidal vector for tumor immunotherapy was advanced with the development of a new vector, Listeria monocytogenes recombinase-induced intracellular death ( $L m$-RIID). This strain features Aduro's live-attenuated double deletion $\mathrm{Lm}$ vaccine (LADD) that has been further modified by the introduction of loxP sites adjacent to essential genes, as well as a gene for Cre recombinase that is inserted under the act $A$ locus (78). In bacterial growth media, $L m$-RIID proliferates and functions normally, however, once it infects host cells and gains entry to the cytosol, Cre-recombinase is produced and excises the essential genes flanked by loxP sites. The loss of these essential genes ultimately results in loss of viability for the bacterium, while still allowing sufficient time for it to produce and secrete the necessary tumor antigens within the cytosol. This construct has demonstrated efficacy similar to the LADD platform in mouse tumor models, inducing a robust anti-tumor immune response, and a substantial reduction in tumor burden. In combination with anti-PD-1 therapy, it conferred maximal protection against tumor growth and regression in a lung metastasis model. Perhaps the most interesting characteristic about this platform is its excellent safety profile. Although the enhanced attenuation of $L m$-RIID did result in reduced immunogenicity in direct comparison to existing platforms, it was shown to be more immunogenic than the killed but metabolically active (KBMA) strain and similarly effective to the live, attenuated, double-deleted (LADD) platform (78). Of note, also, is the recombinant suicidal Listeria monocytogenes strain ( $r s \Delta 2$ ) described by Sinha et al. (77) that undergoes autolysis upon entry into the cytosol but produces and delivers both protein antigen and a eukaryotic expression vector encoding the same antigen to an infected cell. This novel suicidal $L m$ vaccine induced both humoral and cell cytolytic responses to the model antigen, ovalbumin, when delivered both intramuscularly and orally. Importantly, delivery of only the eukaryotic expression plasmid encoding for the antigen by $r s \Delta 2$ resulted in blunted responses compared to the vector that delivered $L m$-produced protein antigen and the eukaryotic expression vector. These novel suicidal $L m$-based vaccine vectors demonstrate that, while improvements may still be desired to match their anti-tumor efficacy to previouslydeveloped live $L m$-based vectors, their effectiveness when given orally, enhanced level of safety, and their greater level of versatility to deliver both protein and nucleic acid cargo demonstrates great promise for them as clinical vectors for tumor immunotherapy.

\section{Listeria as a Targeted Radionuclide Therapeutic (TRT) Platform}

The combination of $L m$-based immunotherapy with radiotherapy has shown promise as a synergistic treatment regimen in a preclinical model for melanoma (86). However, there are still challenges to discover the most efficient sequence of treatment administration for both the $L m$ vaccine and radiotherapy, i.e. concurrently or sequentially, and the offtarget effects of radiotherapy on healthy tissue may complicate the timing and number of treatments $(87,88)$. To overcome these challenges, a recent study utilized a previously engineered $L m$-based vaccine platform that specifically homes to and replicates within tumors as a vector for tumor-specific delivery of radiotherapy (29). Targeted radionuclide therapeutics (TRT) have shown potential in clinical trials as observed by various radionuclide/antibody conjugates that have been effective in treatment of malignancies such as glioblastoma (89) To accomplish this, a tumor-trophic $L m$-based vector was incubated with anti-Listeria antibodies labeled with radionuclide, ${ }^{188} \mathrm{Re}$, thereby creating a tumor-targeting radiotherapy platform (30). This generated a radioactive attenuated $L m$ strain with high viability, stability, and infectivity. When this radioactive $L m$-based therapy was administered in a murine pancreatic cancer model, radioactive ${ }^{188} \mathrm{Re}$ specifically accumulated in the tumors and metastatic lesions, further confirming the ability of $L m$ to act as a tumor-specific vector. Interestingly, while there was accumulation of this radioactive $\mathrm{Lm}$ in both kidney and liver, no histopathological damage and change in liver function was observed one week after the last treatment, suggesting that the highly proliferative tumor cells in metastases are more susceptible to the consequences of radiation-induced DNA damage than normal tissues (30).

However, a limitation of this strategy is the difficulty in generating this antibody dependent $\mathrm{Lm}$ radionuclide construct. This limitation was overcome in a more recent strategy that utilized radioactive phosphate ${ }^{32} \mathrm{P}$ to generate a novel TRT $\mathrm{Lm}$ construct. This simple but elegant method involved starving the attenuated $\mathrm{Lm}$ in saline followed by culturing in media supplemented with ${ }^{32} \mathrm{P}$ (29). The simplicity of this method allows for greater reproducibility and, more importantly, generation of an $\mathrm{Lm}$ construct that is more viable and stable without losing the incorporated radionuclide. This $L m$-TRT also specifically homed to the tumor and metastatic sites in a pancreatic cancer model while demonstrating more effectiveness than its precursor. Interestingly, the major side effect associated with the use of ${ }^{32} \mathrm{P}$ in cancer therapy, accumulation in the bone marrow, was not seen with $L m-{ }^{32} \mathrm{P}$ used as a TRT. As this approach has only been assessed preclinically in a difficult-totreat pancreatic cancer model, application of this technology to additional cancer models that are more amenable to immunotherapy may provide even more promising results.

\section{Incorporation of $\mathbf{L m}$-Derived Products Into Nanovaccine Platforms}

As development continues in safer and more effective live Lmbased vaccines, other groups have focused on utilizing specific products or proteins from Listeria monocytogenes to improve the immunogenicity of nanoparticle-based vaccine platforms (90, 91). Much like $L m$, nanoparticle-based vaccines, comprised of liposomes and/or metal particles, have the ability to selectively deliver cargo to professional antigen presenting cells, and 
accumulate within primary and metastatic tumors (92). In fact, a recently developed nanovaccine utilized the adjuvant properties of LLO to enhance the efficacy of a gold nanoparticle-based vaccine in preclinical development for metastatic melanoma (90). Gold nanoparticles are utilized in tumor nanovaccines due to their small size, ability to disseminate widely throughout the tissues, and ability to specifically accumulate at tumor sites (93). The gold nanoparticle-based vaccine, GNP$\mathrm{LLO}_{91-99}$, was constructed by incorporating gold nanoparticles with $\beta$-D glucose, which increases tumor oxidative stress, and a $\mathrm{H} 2 \mathrm{k}^{\mathrm{d}}$-restricted epitope peptide derived from LLO, LLO ${ }_{91-99}$. GNP-LLO $_{91-99}$ induced a robust production of inflammatory cytokines, a reduction in the intratumoral Treg and MDSC populations, and increased infiltration of LLO and tumorspecific $\mathrm{CD}^{+} \mathrm{T}$ cells into the tumor. Surprisingly, GNP$\mathrm{LLO}_{91-99}$ was more effective at reducing melanoma tumor burden as a monotherapy, than ICI therapy. However, the combination of GNP-LLO ${ }_{91-99}$ with anti-PD-1 and anti-CTLA4 antibodies resulted in synergistic efficacy and complete tumor regression (90) Of note, the melanoma model in this study, $\mathrm{B} 16 \mathrm{~F} 10$, is performed in the $\mathrm{H} 2^{\mathrm{b}}$-restricted C57BL/6 mice while the LLO-derived peptide within GNP-LLO ${ }_{91-99}$ is well characterized as an $\mathrm{H} 2 \mathrm{~K}^{\mathrm{d}}$-restricted LLO epitope suggesting further investigation is warranted to fully understand its mechanism(s) of adjuvancy.

Another utilization of $L m$-derived components in a nanovaccine involves the use of purified LLO in combination with liposomal nanoparticles (91). While liposomes are effective carriers of antigens for delivery to APCs (94), it was hypothesized that the lytic properties of LLO may allow for improved cytosolic release of liposomal cargo after uptake. The LLO-liposome nanoparticle-based vaccine consisted of a liposome, loaded with recombinant OVA protein, as a model tumor antigen, and recombinant LLO (91). When compared against a liposomal nanoparticle-based vaccine containing only ovalbumin, the incorporation of LLO into the nanovaccine resulted in enhanced presentation of the immunodominant CTL epitope, SIINFEKL, by APCs, improved OVA-specific cytolytic and humoral responses, a dramatic delay in growth of OVA-expressing melanoma tumors, and improved survival.

While the different types of nanovaccines have widely divergent compositions and chemistry, the incorporation of $L m$-based components appears to dramatically improve their immunogenicity and anti-tumor efficacy $(90,91)$. These studies suggest that the utilization of $L m$ for tumor immunotherapy may not necessitate the need for the whole organism but just purified listerial components with unique properties to eventuate a safe but effective anti-tumor response.

\section{Listeria as a Gene Delivery Vector}

Due to the cellular tropism of $L m$ and its ability to gain entry to the cytosol after infection, it is uniquely capable as a vector for gene delivery $(84,95,96)$. In a process called bactofection, bacteria can be engineered to deliver eukaryotic expression vectors containing genes encoding for enzymes or protein antigens to an infected cell (97). In fact, previous studies have found Listeria monocytogenes to be a suitable vector for bactofection $(84,96,98)$. Souders et al. performed the first demonstration that this ability of $L m$ to perform bactofection could be utilized in tumor immunotherapy (79). In this study, an $L m$-based bactofection construct platform was made by engineering $L m$ to include a suicide cassette that expresses a phage lysin under the control of the actA promoter, and a eukaryotic expression system containing the HPV16 E7 tumor antigen. Upon infection of APCs, the $L m$ bactofection construction, $L m$-V2, escaped into the cytosol and underwent autolysis while delivering the E7 expression vector. $L m-\mathrm{V} 2$ allowed for proficient expression of the E7 tumor antigen and induced E7-specific CTLs that infiltrated E7-expressing tumors and delayed tumor growth. While $L m-\mathrm{V} 2 \mathrm{did}$ not produce as robust an anti-tumor response as the $L m$-LLO-E7 vector that secreted E7 protein in the cytosol and did not undergo autolysis, it served as a proof-of-concept for a strategy that would ensure safety as a primary feature (79). Schoen et al. also demonstrated the utility of $L m$ as a vector for bactofection of antigens in a study where, instead of a CMV-driven expression vector, they introduced mRNA encoding for OVA (99). Upon infection of APCs in vitro, this vector was able to facilitate the activation of OVA-specific CTLs. While no in vivo experiments were performed in this study, they also demonstrated the ability of $L m$ to deliver nucleic acids to tumor cells upon infection. This strategy of utilizing $\mathrm{Lm}$ as a vector for bactofection of tumor cells was furthered by work from Pijkeren et al. that described the development of an $\mathrm{Lm}$-based bactofection vector engineered to release a eukaryotic expression plasmid encoding luciferase subsequent to antibiotic administration (100, 101). In vitro infection and intratumoral infection in vivo of this bactofection construct did result in robust luciferase expression after antibiotic treatment suggesting $L m$ may also find utility as a tumor-selective delivery vector for nucleic acids.

While $L m$ is certainly capable as a vector for gene therapy to deliver nucleic acid cargo and facilitate protein expression in a target cell, current constructs are still not as effective at delivering antigen than $\mathrm{Lm}$ constructs that encode and secrete protein directly (102). Thus, since this would limit the ability to maximally present antigens, the ability of these various constructs to elicit robust antigen-specific $\mathrm{CD} 8^{+}$and $\mathrm{CD} 4^{+} \mathrm{T}$ cell responses is currently limited and this technology will likely require further development prior to its successful entry into clinical trials.

\section{COMBINATION THERAPIES WITH LM}

Lm-based vaccines alone stimulate robust immune responses, increase immune infiltrates into tumors, and result in durable anti-tumor responses in many preclinical models of cancer (31). However, the heterogeneity of tumors and the TME in humans remains a major obstacle in obtaining effective responses to many cancer therapeutics in the broader population (103). One approach to overcome the detrimental effect of tumor heterogeneity on drug sensitivity is to utilize a combination therapy approach that consists of multiple drugs with 
complementary mechanisms of action (104). We describe in detail below combination therapy approaches utilizing $L m$-based vaccines that have shown promise in treatment of cancers in the clinic and preclinically.

\section{Lm in Combination With Immune Checkpoint Inhibitors (ICls)}

One mechanism by which a tumor can escape immune-mediated destruction is by enhancing the expression of immune checkpoint molecules on $\mathrm{T}$ cells such as PD-1 and CTLA-4 (105). While effector $\mathrm{T}$ cells can transiently express these molecules during activation or prolonged activation, high levels of these molecules are associated with an exhausted $\mathrm{T}$ cell phenotype, thereby limiting their tumor lytic potential (106), Since the anti-tumor efficacy of $L m$-based vaccines is through induction of potent tumor-specific $\mathrm{CD}^{+} \mathrm{T}$ cells, maintaining their functionality is necessary for an effective immunotherapeutic platform (3). In fact, blockading antibodies that act as immune checkpoint inhibitors (ICI) dramatically enhance the functionality of anti-tumor $\mathrm{T}$ cell responses, an attribute that has revolutionized the treatment of various solid tumors $(105,107)$. Therefore, combination of these agents with $\mathrm{Lm}$-based immunotherapeutics, which are able to induce potent and effective CTLs that infiltrate the TME may lead to even greater survival in cancer patients.

Thus far, various preclinical studies have found a synergistic effect in the combination of $L m$-based therapeutics with ICIs (108-111). The initial study demonstrating synergy between anti-PD-1 blockading antibodies and the anti-tumor efficacy of $L m$-based vaccines was by Mkrtichyan et al. (110). This study utilized the well-characterized $L m$-LLO-E7 vaccine and assessed the ability of ICI to improve anti-tumor responses against the TC-1 tumor model for HPV-associated cancers. While they did find a synergistic anti-tumor effect by combining $L m$-LLO-E7 with anti-PD-1 antibody, the dosage of Lm-LLO-E7 was approximately 5-fold lower and less effective than previous studies which may have better revealed the synergistic effect of these two therapeutics (110). Further support for this finding was found in a study assessing the efficacy of an $\mathrm{Lm}$-based vaccine in combination with PD-1 blockade in a preclinical model of pancreatic ductal adenocarcinoma (PDAC) (109). PDAC is a highly heterogeneous solid tumor that is categorized as a "cold" tumor with limited immune cell tumor infiltrates making it less receptive to ICI treatment alone. However, utilizing an $L m$-based construct expressing Annexin A2 ( $L m$-ANXA2), a relevant PDAC tumor antigen, Kim et al., demonstrated that sequential treatment with Lm-ANXA2 followed by PD-1 blockade resulted in an increase in overall survival of PDAC-bearing mice compared with either Lm-ANXA2 or anti-PD-1 therapy alone. Moreover, this therapeutic strategy elicited strong ANXA2specific immune responses in the TME as evidenced by increase production of IFN $\gamma$, an observation not found in the group receiving anti-PD-1 alone (109) In addition to PDAC and $\mathrm{HPV}$-associated cancers, $L m$-based vaccines have also been reported to synergize with ICI in a particularly challenging malignancy with low survival rates, hepatocellular carcinoma
(HCC). In fact, unlike many other cancers, ICI has limited efficacy and can even lead to hyperprogression in some patients $(112,113)$. Interestingly, an $L m$-based vaccine expressing a multivalent HCC tumor antigen, Lmdd-MPFG, can induce PD-L1 expression, the ligand for PD-1, in HCC tumor cells suggesting that it may synergize with ICI therapy (108). When Lmdd-MPFG was administered along with antiPD-1 antibody, it resulted in significantly reduced tumor burden as compared to PD-1 blockade or $L m$ vaccine treatment alone. While Lmdd-MPFG alone did result in significant anti-tumor efficacy, PD-1 blockade alone did not, mirroring clinical experience, and suggesting that the $L m$-based vaccine sensitized HCC to ICI. Further evidence suggested that the ability of Lmdd-MPFG to polarize TAMs to the M1 phenotype played a role in the sensitization of HCC to PD-1 blockade (108). In addition to ICI synergizing with $L m$-based vaccines in a therapeutic setting, it has also been found to enhance protection against tumor challenge in an murine model of melanoma expressing ovalbumin, B16F10-OVA (111). While administration of the $L m$-based vaccine expressing $\mathrm{OVA}_{257-264}$ fused to ActAN100, Lm-OVA, provided significant protection against subsequent challenge with B16F10-OVA, up to $30 \%$ of mice developed tumors. The authors hypothesized that this could be due to the induction of peripheral tolerance mechanisms such as immune checkpoints that allow tumor escape. Therefore, approximately 2 months after $\mathrm{Lm}$-based vaccination, mice were challenged with tumor, and received ICI (either anti-PD-1, anti-CTLA-4, or anti-PD-L1) every 10 days subsequently, in order to maintain anti-tumor $\mathrm{T}$ cell function. Each of the ICI antibodies enhanced the anti-tumor effect of Lm-OVA. More strikingly, there was significantly more mice remaining tumor-free after the addition of checkpoint blockade compared to vaccination with $L m$-OVA alone. The data from these studies suggest that the efficacy of $L m$ vaccines can be enhanced by ICI therapy but $L m$ vaccination can also sensitize previously "cold" tumors, such as PDAC, to the powerful potential of ICI therapy $(108,111)$.

Use of checkpoint inhibitors in combination with $L m$ therapy is also being currently evaluated in various phases of clinical trials (Table 1). As we discussed in section 1.3.2, a Phase II study has demonstrated the positive immunomodulatory effect of the combination therapy of CRS-207 ( $\mathrm{Lm}$-mesothelin) with nivolumab (anti-PD-1) on the TME in the treatment of human PDAC (56).

\section{Lm Combination With Adoptive Cell Therapy (ReACT Cells)}

Adoptive cell therapy (ACT) is the use of engineered or naturally occurring immune cells for the treatment of cancer (114). This strategy has enjoyed unprecedented success in oncotherapy, with high response rates in hematopoietic malignancies and melanoma (114). However, this strategy comes with serious limitations and drawbacks that make its use limited in the therapy of solid tumors (115). One of the most significant challenges is that the TME is an immunosuppressive environment with elevated levels of anti-inflammatory 
cytokines, inhibitory receptor ligands, and immunosuppressive cell types such as MDSCs that impede the activation, proliferation and differentiation of anti-tumor immune cells (116). Due to their ability to modulate the TME and reduce peripheral tolerance mechanisms, $L m$-based vaccines may be uniquely able to overcome the limitations of this therapeutic strategy and enhance the efficacy of ACT (50). While Lm improves the ability of immune cells to infiltrate into the TME, $L m$ is also able to infect MDSCs which specifically home to the TME and likely mitigate immunosuppression within the tumor (117). Xin et al., has developed an approach that utilizes $L m$-based vaccines to enhance ACT and overcome the immunosuppressive TME in a strategy named Reenergized ACT (ReACT). To advance their work, they utilized monospecific and dual-specific $\mathrm{CD}^{+} \mathrm{T}$ cells that recognize a tumor antigen, gp100, or both a tumor antigen and an $\mathrm{Lm}$-derived antigen, OVA, respectively. After allowing for tumors to establish, mice were administered mono or dual-specific $\mathrm{CD}^{+}$ $\mathrm{T}$ cells followed by intratumoral injection of the $\mathrm{Lm}$-OVA vaccine. Surprisingly, mice receiving only CTLs did not respond and less than $10 \%$ of mice receiving only tumorspecific CTLs and i.t. Lm-OVA responded, In contrast, 69\% of mice receiving both i.t. $L m$-OVA injection and dual-specific CTLs that recognize the tumor and $L m$-derived antigens responded and eradicated their tumors. This method also resulted in greater tumor infiltration and function of the transferred CTLs and reduced expression of immune checkpoint molecules by CTLs. In the TME, MDSCs and Tregs made up a lower percentage of overall tumor cells in each of the $\mathrm{Lm}$-treated groups but only the dual-specific CTLs with $\mathrm{Lm}$ treatment group had a higher Teff/Treg ratio and lower absolute Tregs than other treatment groups $(14,15)$. As loss of the targeted antigen is associated with therapeutic resistance in ACT, broadening of the anti-tumor response through epitope spreading to additional tumor antigens should result in a more durable anti-tumor response $(118,119)$. In fact, this therapeutic strategy did lead to epitope spreading that induced long-lasting endogenous memory cells that provided protection against subsequent challenge (14). In this preclinical model of melanoma, the only adverse events associated with ReACT was limited to mild vitiligo at the primary tumor injection site (14).

\section{Lm in Heterologous Vaccination Schedules}

Heterologous prime boost involves the administration of the same antigens using different vaccine vector platforms to generate a more robust immune response than a homologous prime boost vaccine regimen $(120,121) . \mathrm{Lm}$-based vaccines are particularly effective at generating robust responses when used as a booster or primer in combination with various vaccine vectors including viruses, DNA and peptides (122-125). In a preclinical study that involved the use of a DNA vaccine encoding the prostate-specific antigen prostatic acid phosphatase (PAP) along with an attenuated $L m$ vaccine, LADD-PAP, greater antitumor efficacy was seen in the prime boost regimen as compared to the DNA vaccine only regimen or the LADD-PAP only regimen
(122). This heterologous vaccination schedule interestingly led to the induction of more $\mathrm{CD} 4^{+} \mathrm{T}$ cells, which may have played a role in the enhanced antitumor immune response. Enhanced cellular immune responses were observed with a heterologous prime boost regimen consisting of an EGFRvIII peptide vaccine, PEPvIII, and an Lm-based vaccine targeting EGFRvIII, LmEGFRvIIIx5, which resulted in greater induction of EGFRspecific $\mathrm{CD}^{+} \mathrm{T}$ cells than either vaccine alone (125). Interestingly, this enhanced EGFR-specific CTL response was only observed when the Lm-EGFRvIIIx 5 was given as a booster to a previous peptide immunization. $L m$-based vaccines have also been found to boost anti-tumor immunity when delivered in heterologous prime boost regimens with viral vectors $(123,124)$. In a prophylactic study, mice receiving a modified vaccinia Ankara vaccine expressing human p53, MVA-p53, followed by an $L m$-based vaccine expressing human p53, LmddA-LLO-p53, resulted in greater anti-tumor protection against the murine breast cancer model, 4T1p53 (124). Further, the use of an Lmbased vaccine targeting the model tumor antigen chicken ovalbumin, Lm-OVA, prior to boosting with an oncolytic Maraba virus, MRB-OVA, resulted in a greater reduction in B16F10-OVA melanoma tumor growth, more tumor-free mice, and greater overall survival than mice receiving an adenovirusbased vaccine as the priming agent (123). Most importantly, this incorporation of $\mathrm{Lm}$-based vaccines into heterologous prime boost approaches for cancer immunotherapy has already been found effective in a clinical trial. As we previously discussed, in a Phase II trial in PDAC patients, CRS-207 ( $\mathrm{Lm}$-mesothelin) in combination with Cy/GVAX improved median overall survival and enhanced induction of mesothelin specific $\mathrm{CD}^{+} \mathrm{T}$ cells as compared to Cy/GVAX alone (53).

\section{Lm in Combination With Radiotherapy}

Radiation therapy (RT) has been a mainstay therapeutic option for nearly a century in the treatment of various forms of malignancies (126). However, recent work has found that RT does not solely disrupt tumor cell division but has immunostimulatory effects in the TME (127). RT helps trigger the release of tumor antigens, improve presentation of tumor antigens to T cells, and induces an inflammatory response in the TME that results in elevated IFN $\gamma$ levels and reduced immunosuppression $(86,128)$. However, despite all these attractive features of $\mathrm{RT}$, resistance and recurrence still occur through multiple mechanisms (129). In order to improve the efficacy of $\mathrm{RT}$, a recent study utilized a combination of RT and Lm-OVA in the B16-OVA melanoma model (86). This approach resulted in an increase in the activated $\mathrm{T}$ cells infiltrating the TME and a more robust response in the combination therapy than in the use of $L m$ or RT alone. One possible explanation for the observed synergy was that both the $L m$ vaccine and the RT seem to activate different components of the immune system (86). A similar synergistic effect of RT and $L m$-based vaccines was observed in a murine model of prostate cancer. In this study, an $L m$-based vaccine expressing human prostate antigen (PSA) in combination with RT resulted in synergistic induction of PSA-specific splenic $\mathrm{T}$ cell responses and therapeutic anti-tumor efficacy (130). 
In an effort to improve on the efficacy and specific delivery of the lethal radiation therapy to the tumors and metastasis, the Gravekamp group has developed an elegant method to use $L m$ as a delivery vector for radioisotopes directly to tumors. In this study using $L m$ infused with ${ }^{32}$ Phosphorous $\left(L m-{ }^{32} \mathrm{P}\right)$, this group was able to demonstrate that $\mathrm{Lm}^{3}{ }^{32} \mathrm{P}$ specifically homes to the TME in a mouse model for PDAC (29). The DNA damage caused by the radioisotope coupled with the antitumor efficacy of $\mathrm{Lm}$ itself makes this platform very potent and promising in cancer therapy.

\section{Lm in Combination With Inhibitors of Complement Signaling}

The complement cascade is well characterized to play a crucial role in the innate immune response to pathogens (131). However, evidence over the past decade demonstrates that the complement pathway and its components can also regulate adaptive immunity, particularly anti-tumor $\mathrm{T}$ cell responses (132). In fact, complement is highly activated in tumors and this activation results in $\mathrm{T}$ cell dysfunction and blunted antitumor responses (133). Importantly, a small molecule inhibitor of the C5a receptor (C5aR1), PMX53, enhances infiltration and function of tumor-infiltrating $\mathrm{T}$ cells leading to tumor regression in mouse cancer models (134). Therefore, inhibition of complement signaling may make the TME more amenable to anti-tumor T cells and synergize with an $L m$-based vaccine. In a recent study, this synergistic potential was confirmed by demonstrating that treatment with PMX53 synergized with tumor vasculature targeting $L m$-based vaccines to significantly reduce primary tumor growth and reduce lung metastases in a murine model of metastatic breast cancer, 4T1 (135). Furthermore, this synergistic potential was correlated with reduced levels of MDSCs and Tregs in the lungs of tumorbearing mice and enhanced maturation of lung-associated dendritic cells.

\section{Lm in Combination With Traditional Chemotherapy}

Chemotherapy remains the gold standard for the treatment of various forms of cancers. Chemotherapy may also synergize with and augment the activity of immunotherapies, such as $L m$-based vaccines, by inducing direct cell death that results in release and presentation of tumor antigens and maturation of dendritic cells $(136,137)$. Chemotherapy, such as low-dose cyclophosphamide, can also significantly reduce the Treg population in the TME (138). In fact, in a murine model of hepatic metastases, animals treated with attenuated $L m$ along with low dose cyclophosphamide had reduced levels of Tregs in the TME and spleen and a dramatic increase in overall survival (139). The Tregdepleting properties of cyclophosphamide were also found to enhance the efficacy of an $L m$-based vaccine in the challenging KPC mouse model for PDAC $(140,141)$. In this study, Treg depletion by cyclophosphamide was further enhanced by addition of anti-CD25 depleting antibody in combination with an $L m$-based vaccine targeting a 25 amino acid region of $\mathrm{Kras}^{\mathrm{G} 12 \mathrm{D}}$,
LM-Kras. In KPC mice less than 2 months old, the combination of LM-Kras and cyclophosphamide/anti-CD25 was found to significantly delay progression of precancerous pancreatic lesions to PDAC and enhance survival in comparison to LMKras or cyclophosphamide/anti-CD25 alone. T cell infiltration and Th1 responses in the pancreas were also enhanced in the group receiving LM-Kras and cyclophosphamide/anti-CD25 in comparison to LM-Kras alone. While it is not possible to dissect the exact contribution of cyclophosphamide in the efficacy of LMKras in this study, much like another PDAC study that included cyclophosphamide in all treatment groups (109). These results provide additional pre-clinical evidence that chemotherapy will likely have an important role to play in effective treatment regimens with $L m$-based vaccines. In humans, several clinical trials have incorporated chemotherapeutic agents, such as cyclophosphamide, pemetrexed and cisplatin, along with $\mathrm{Lm}$ based vaccines into their treatment regimen $(41,44,53,55,56$, 60). As yet, evidence from these clinical trials suggest some chemotherapy regimens may provide a clinical benefit in combination with $L m$-based vaccines $(53,60)$ while others have found mixed or negligible benefit from this combination (41).

\section{Conclusion}

The recent results reported from several clinical trials demonstrate the promising future of $L m$-based tumor immunotherapies but also reveal challenges that educate the future development of the platform $(41,42,60)$. Numerous improvements to the platform have already been reported in preclinical studies that would not be evident in the clinical results as they are mostly utilizing first-generation $L m$-based constructs $(29,30,78)$. Even with the use of these first generation constructs, promising clinical responses have been reported in several cancers including cervical cancer, malignant pleural mesothelioma, and canine osteosarcoma (74). As results from clinical and preclinical studies demonstrate, improvements to therapeutic efficacy may be realized utilizing $L m$-based vaccines in heterologous prime boost regimens with other vectors and by combination with synergistic therapeutic strategies such as ACT and ICI $(14,53,108,110,122,123,134)$. However, there still remain several challenges going forward to realize the full potential of Lm-based vaccines. As a recent clinical trial suggests, $L m$-based vaccines may not significantly benefit from combination with particular chemotherapies that suppress immunity and increase risk of adverse events (41). Further, while rarely observed in clinical trials as yet, the incidence of listeriosis in humans and dogs may justify additional study into the variables contributing to this risk $(43,76)$. Nevertheless, improvements to clinical safety may be realized with the adoption of recently developed suicide strains, such as $\mathrm{Lm}$-RIID, that would dramatically reduce the risk of listeriosis (78). As with other current vaccine platforms, treatment resistance due to immune escape will also likely be an ongoing challenge, particularly due to many of the current vaccines targeting a single tumor antigen (142, 143). While not yet published, improvements to the antigens targeted by $\mathrm{Lm}$-based vaccines such as the multivalent ADXS-HOT platform targeting 
immunogenic hotspot mutations and the patient-personalized ADXS-NEO platform will likely bring improvements in efficacy, in part by limiting immune escape. Finally, recent advancements in leveraging its tumor-trophic potential suggest that attenuated Lm-based therapeutics can provide multiple separate but effective anti-tumor mechanisms which, if fully leveraged, may also mitigate therapeutic resistance $(14,15,29,30,117,144)$. With our improved understanding of its clinical performance and the continued development of the platform, the future is promising for $L m$-based therapeutics.

\section{REFERENCES}

1. Kantoff PW, Higano CS, Shore ND, Berger ER, Small EJ, Penson DF, et al. Sipuleucel-T immunotherapy for castration-resistant prostate cancer. N Engl J Med (2010) 363(5):411-22. doi: 10.1056/NEJMoa1001294

2. Hollingsworth RE, Jansen K. Turning the corner on therapeutic cancer vaccines. NPJ Vaccines (2019) 4:7. doi: 10.1038/s41541-019-0103-y

3. Gunn GR, Zubair A, Peters C, Pan ZK, Wu TC, Paterson Y. Two Listeria monocytogenes vaccine vectors that express different molecular forms of human papilloma virus-16 (HPV-16) E7 induce qualitatively different T cell immunity that correlates with their ability to induce regression of established tumors immortalized by HPV-16. J Immunol (2001) 167 (11):6471-9. doi: 10.4049/jimmunol.167.11.6471

4. Didot A. Essai sur la prophylaxie du cancer par la syphilization artificielle. Bull Acad Roy Belge (1851) 1852(11):100-72.

5. Hall SS. A commotion in the blood: life, death, and the immune system. 1st ed Vol. xiv. . New York: Henry Holt (1997). 544 p.

6. Oelschlaeger TA. Bacteria as tumor therapeutics? Bioeng Bugs (2010) 1 (2):146-7. doi: 10.4161/bbug.1.2.11248

7. Busch W. Aus der Sitzung der medicinischen Section vom 13 November 1867. Berl Klin Wochenschr (1868) 5:137.

8. Fehleisen F. Ueber die Züchtung der Erysipelkokken auf künstlichem Nährboden und ihre Übertragbarkeit auf den Menschen. Dtsch Med Wochenschr (1882) 8(31):553-4. doi: 10.1055/s-0029-1196806

9. Coley WB. The Diagnosis and Treatment of Bone Sarcoma. Glasgow Med J (1936) 126(2):49-86.

10. McCarthy EF. The toxins of William B. Coley and the treatment of bone and soft-tissue sarcomas. Iowa Orthop J (2006) 26:154-8.

11. Coley WBII. Contribution to the Knowledge of Sarcoma. Ann Surg (1891) 14 (3):199-220. doi: 10.1097/00000658-189112000-00015

12. Durgeau A, Virk Y, Corgnac S, Mami-Chouaib F. Recent Advances in Targeting CD8 T-Cell Immunity for More Effective Cancer Immunotherapy. Front Immunol (2018) 9:14. doi: 10.3389/fimmu.2018.00014

13. Dunn GP, Bruce AT, Ikeda H, Old LJ, Schreiber RD. Cancer immunoediting: from immunosurveillance to tumor escape. Nat Immunol (2002) 3(11):9918. doi: 10.1038/ni1102-991

14. Xin G, Khatun A, Topchyan P, Zander R, Volberding PJ, Chen Y, et al. Pathogen-Boosted Adoptive Cell Transfer Therapy Induces Endogenous Antitumor Immunity through Antigen Spreading. Cancer Immunol Res (2020) 8(1):7-18. doi: 10.1158/2326-6066.CIR-19-0251

15. Xin G, Schauder DM, Jing W, Jiang A, Joshi NS, Johnson B, et al. Pathogen boosted adoptive cell transfer immunotherapy to treat solid tumors. Proc Natl Acad Sci U S A (2017) 114(4):740-5. doi: 10.1073/pnas.1614315114

16. Overwijk WW, Theoret MR, Finkelstein SE, Surman DR, de Jong LA, VythDreese FA, et al. Tumor regression and autoimmunity after reversal of a functionally tolerant state of self-reactive CD8+ T cells. J Exp Med (2003) 198(4):569-80. doi: 10.1084/jem.20030590

17. Minton NP. Clostridia in cancer therapy. Nat Rev Microbiol (2003) 1 (3):237-42. doi: 10.1038/nrmicro777

18. Barbé S, Van Mellaert L, Theys J, Geukens N, Lammertyn E, Lambin P, et al. Secretory production of biologically active rat interleukin-2 by Clostridium acetobutylicum DSM792 as a tool for anti-tumor treatment. FEMS Microbiol Lett (2005) 246(1):67-73. doi: 10.1016/j.femsle.2005.03.037

\section{AUTHOR CONTRIBUTIONS}

MO, drafting. YP, revision. LW, drafting and revision. All authors contributed to the article and approved the submitted version.

\section{FUNDING}

National Institutes of Health 1R15CA216205-01 (LW).

19. Wood LM, Guirnalda PD, Seavey MM, Paterson Y. Cancer immunotherapy using Listeria monocytogenes and listerial virulence factors. Immunol Res (2008) 42(1-3):233-45. doi: 10.1007/s12026-008-8087-0

20. Agrawal N, Bettegowda C, Cheong I, Geschwind J-F, Drake CG, Hipkiss EL, et al. Bacteriolytic therapy can generate a potent immune response against experimental tumors. Proc Natl Acad Sci U S A (2004) 101(42):15172-7. doi: 10.1073/pnas.0406242101

21. Mengaud J, Chenevert J, Geoffroy C, Gaillard JL, Cossart P. Identification of the structural gene encoding the $\mathrm{SH}$-activated hemolysin of Listeria monocytogenes: listeriolysin $\mathrm{O}$ is homologous to streptolysin $\mathrm{O}$ and pneumolysin. Infect Immun (1987) 55(12):3225-7. doi: 10.1128/ IAI.55.12.3225-3227.1987

22. Poussin MA, Goldfine H. Involvement of Listeria monocytogenes phosphatidylinositol-specific phospholipase $\mathrm{C}$ and host protein kinase $\mathrm{C}$ in permeabilization of the macrophage phagosome. Infect Immun (2005) 73 (7):4410-3. doi: 10.1128/IAI.73.7.4410-4413.2005

23. Tilney LG, Portnoy DA. Actin filaments and the growth, movement, and spread of the intracellular bacterial parasite, Listeria monocytogenes. J Cell Biol (1989) 109(4 Pt 1):1597-608. doi: 10.1083/jcb.109.4.1597

24. Brunt LM, Portnoy DA, Unanue ER. Presentation of Listeria monocytogenes to CD8+ T cells requires secretion of hemolysin and intracellular bacterial growth. J Immunol (1990) 145(11):3540-6. doi: 10.1084/jem.175.6.1531

25. Tufail S, Badrealam KF, Owais M, Zubair S. Illuminating the Petite Picture of T Cell Memory Responses to Listeria monocytogenes. BioMed Res Int (2013) 2013:121684. doi: 10.1155/2013/121684

26. Orme IM. Active and memory immunity to Listeria monocytogenes infection in mice is mediated by phenotypically distinct $\mathrm{T}$-cell populations. Immunology (1989) 68(1):93-5.

27. D'Orazio SEF. Innate and Adaptive Immune Responses during Listeria monocytogenes Infection. Microbiol Spectr (2019) 7(3):21-2. doi: 10.1128/ microbiolspec.GPP3-0065-2019

28. Stark FC, Sad S, Krishnan L. Intracellular Bacterial Vectors That Induce CD8 (+) T Cells with Similar Cytolytic Abilities but Disparate Memory Phenotypes Provide Contrasting Tumor Protection. Cancer Res (2009) 69 (10):4327. doi: 10.1158/0008-5472.CAN-08-3160

29. Chandra D, Selvanesan BC, Yuan Z, Libutti SK, Koba W, Beck A, et al. 32 Phosphorus selectively delivered by listeria to pancreatic cancer demonstrates a strong therapeutic effect. Oncotarget (2017) 8(13):2072940. doi: 10.18632/oncotarget.15117

30. Quispe-Tintaya W, Chandra D, Jahangir A, Harris M, Casadevall A, Dadachova E, et al. Nontoxic radioactive Listeria(at) is a highly effective therapy against metastatic pancreatic cancer. Proc Natl Acad Sci U S A (2013) 110(21):8668-73. doi: 10.1073/pnas.1211287110

31. Wood LM, Paterson Y. Attenuated Listeria monocytogenes: a powerful and versatile vector for the future of tumor immunotherapy. Front Cell Infect Microbiol (2014) 4:51-. doi: 10.3389/fcimb.2014.00051

32. Roden RBS, Stern PL. Opportunities and challenges for human papillomavirus vaccination in cancer. Nat Rev Cancer (2018) 18(4):24054. doi: $10.1038 /$ nrc. 2018.13

33. McCredie MR, Sharples KJ, Paul C, Baranyai J, Medley G, Jones RW, et al. Natural history of cervical neoplasia and risk of invasive cancer in women with cervical intraepithelial neoplasia 3: a retrospective cohort study. Lancet Oncol (2008) 9(5):425-34. doi: 10.1016/S1470-2045(08)70103-7 
34. Gillison ML, Alemany L, Snijders PJ, Chaturvedi A, Steinberg BM, Schwartz S, et al. Human papillomavirus and diseases of the upper airway: head and neck cancer and respiratory papillomatosis. Vaccine (2012) 30(Suppl 5): F34-54. doi: 10.1016/j.vaccine.2012.05.070

35. Pal A, Kundu R. Human Papillomavirus E6 and E7: The Cervical Cancer Hallmarks and Targets for Therapy. Front Microbiol (2019) 10:3116. doi: $10.3389 /$ fmicb. 2019.03116

36. Wallecha A, Wood L, Pan ZK, Maciag PC, Shahabi V, Paterson Y. Listeria monocytogenes-derived listeriolysin $\mathrm{O}$ has pathogen-associated molecular pattern-like properties independent of its hemolytic ability. Clin Vaccine Immunol (2013) 20(1):77-84. doi: 10.1128/CVI.00488-12

37. Wood LM, Pan ZK, Shahabi V, Paterson Y. Listeria-derived ActA is an effective adjuvant for primary and metastatic tumor immunotherapy. Cancer Immunol Immunother (2010) 59(7):1049-58. doi: 10.1007/s00262-0100830-4

38. Peng X, Treml J, Paterson Y. Adjuvant properties of listeriolysin O protein in a DNA vaccination strategy. Cancer Immunol Immunother (2007) 56 (6):797-806. doi: 10.1007/s00262-006-0240-9

39. Lauer P, Hanson B, Lemmens EE, Liu W, Luckett WS, Leong ML, et al. Constitutive Activation of the PrfA regulon enhances the potency of vaccines based on live-attenuated and killed but metabolically active Listeria monocytogenes strains. Infect Immun (2008) 76(8):3742-53. doi: 10.1128/IAI.00390-08

40. Maciag PC, Radulovic S, Rothman J. The first clinical use of a live-attenuated Listeria monocytogenes vaccine: a Phase I safety study of Lm-LLO-E7 in patients with advanced carcinoma of the cervix. Vaccine (2009) 27 (30):3975-83. doi: 10.1016/j.vaccine.2009.04.041

41. Basu P, Mehta A, Jain M, Gupta S, Nagarkar RV, John S, et al. A Randomized Phase 2 Study of ADXS11-001 Listeria monocytogenes-Listeriolysin O Immunotherapy With or Without Cisplatin in Treatment of Advanced Cervical Cancer. Int J Gynecol Cancer (2018) 28(4):764-72. doi: 10.1097/ IGC.0000000000001235

42. Huh WK, Brady WE, Fracasso PM, Dizon DS, Powell MA, Monk BJ, et al. Phase II study of axalimogene filolisbac (ADXS-HPV) for platinumrefractory cervical carcinoma: An NRG oncology/gynecologic oncology group study. Gynecol Oncol (2020) 158(3):562-9. doi: 10.1016/ j.ygyno.2020.06.493

43. Fares E, McCloskey CB, Gutierrez A, Princiotta M, Salinas LJ, Drevets DA. Vaccine strain Listeria monocytogenes bacteremia occurring 31 months after immunization. Infection (2019) 47(3):489-92. doi: 10.1007/s15010-0181249-7

44. Safran H, Leonard KL, Perez K, Vrees M, Klipfel A, Schechter S, et al. Tolerability of ADXS11-001 Lm-LLO Listeria-Based Immunotherapy With Mitomycin, Fluorouracil, and Radiation for Anal Cancer. Int J Radiat Oncol Biol Phys (2018) 100(5):1175-8. doi: 10.1016/j.ijrobp.2018.01.004

45. Seufferlein T, Ettrich TJ. Treatment of pancreatic cancer-neoadjuvant treatment in resectable pancreatic cancer (PDAC). Transl Gastroenterol Hepatol (2019) 4:21. doi: 10.21037/tgh.2019.03.05

46. Royal RE, Levy C, Turner K, Mathur A, Hughes M, Kammula US, et al. Phase 2 trial of single agent Ipilimumab (anti-CTLA-4) for locally advanced or metastatic pancreatic adenocarcinoma. J Immunother (2010) 33(8):82833. doi: $10.1097 /$ CJI.0b013e3181eec14c

47. Blando J, Sharma A, Higa MG, Zhao H, Vence L, Yadav SS, et al. Comparison of immune infiltrates in melanoma and pancreatic cancer highlights VISTA as a potential target in pancreatic cancer. Proc Natl Acad Sci U S A (2019) 116(5):1692-7. doi: 10.1073/pnas.1811067116

48. Teng MW, Ngiow SF, Ribas A, Smyth MJ. Classifying Cancers Based on Tcell Infiltration and PD-L1. Cancer Res (2015) 75(11):2139-45. doi: 10.1158/ 0008-5472.CAN-15-0255

49. Le K, Wang J, Zhang T, Guo Y, Chang H, Wang S, et al. Overexpression of Mesothelin in Pancreatic Ductal Adenocarcinoma (PDAC). Int J Med Sci (2020) 17(4):422-7. doi: 10.7150/ijms.39012

50. Wallecha A, Singh R, Malinina I. Listeria monocytogenes (Lm)-LLO immunotherapies reduce the immunosuppressive activity of myeloidderived suppressor cells and regulatory $\mathrm{T}$ cells in the tumor microenvironment. J Immunother (2013) 36(9):468-76. doi: 10.1186/20511426-1-S1-O18
51. Brockstedt DG, Giedlin MA, Leong ML, Bahjat KS, Gao Y, Luckett W, et al. Listeria-based cancer vaccines that segregate immunogenicity from toxicity. Proc Natl Acad Sci U S A (2004) 101(38):13832-7. doi: 10.1073/ pnas.0406035101

52. Le DT, Brockstedt DG, Nir-Paz R, Hampl J, Mathur S, Nemunaitis J, et al. A live-attenuated Listeria vaccine (ANZ-100) and a live-attenuated Listeria vaccine expressing mesothelin (CRS-207) for advanced cancers: phase I studies of safety and immune induction. Clin Cancer Res (2012) 18(3):85868. doi: 10.1158/1078-0432.CCR-11-2121

53. Le DT, Wang-Gillam A, Picozzi V, Greten TF, Crocenzi T, Springett G, et al. Safety and survival with GVAX pancreas prime and Listeria Monocytogenesexpressing mesothelin (CRS-207) boost vaccines for metastatic pancreatic cancer. J Clin Oncol (2015) 33(12):1325-33. doi: 10.1200/JCO.2014.57.4244

54. Denham JD, Lee DH, Castro M, Pandya S, Aslam S, Nanjappa S, et al. Two cases of disseminated infection following live organism anti-cancer vaccine administration in cancer patients. Int J Infect Dis (2018) 72:1-2. doi: 10.1016/j.ijid.2018.04.004

55. Le DT, Picozzi VJ, Ko AH, Wainberg ZA, Kindler H, Wang-Gillam A, et al. Results from a Phase IIb, Randomized, Multicenter Study of GVAX Pancreas and CRS-207 Compared with Chemotherapy in Adults with Previously Treated Metastatic Pancreatic Adenocarcinoma (ECLIPSE Study). Clin Cancer Res (2019) 25(18):5493-502. doi: 10.1158/1078-0432.CCR-18-2992

56. Tsujikawa T, Crocenzi T, Durham JN, Sugar EA, Wu AA, Onners B, et al. Evaluation of Cyclophosphamide/GVAX Pancreas Followed by Listeriamesothelin (CRS-207) With or Without Nivolumab in Patients with Pancreatic Cancer. Clin Cancer Res (2020) 26:3578-88. doi: 10.1158/10780432.CCR-19-3978

57. Rossini M, Rizzo P, Bononi I, Clementz A, Ferrari R, Martini F, et al. New Perspectives on Diagnosis and Therapy of Malignant Pleural Mesothelioma. Front Oncol (2018) 8:91. doi: 10.3389/fonc.2018.00091

58. Disselhorst MJ, Quispel-Janssen J, Lalezari F, Monkhorst K, de Vries JF, van der Noort V, et al. Ipilimumab and nivolumab in the treatment of recurrent malignant pleural mesothelioma (INITIATE): results of a prospective, single-arm, phase 2 trial. Lancet Respir Med (2019) 7(3):260-70. doi: 10.1016/S2213-2600(18)30420-X

59. Vogelzang NJ, Rusthoven JJ, Symanowski J, Denham C, Kaukel E, Ruffie P, et al. Phase III study of pemetrexed in combination with cisplatin versus cisplatin alone in patients with malignant pleural mesothelioma. J Clin Oncol (2003) 21(14):2636-44. doi: 10.1200/JCO.2003.11.136

60. Hassan R, Alley E, Kindler H, Antonia S, Jahan T, Honarmand S, et al. Clinical Response of Live-Attenuated, Listeria monocytogenes Expressing Mesothelin (CRS-207) with Chemotherapy in Patients with Malignant Pleural Mesothelioma. Clin Cancer Res (2019) 25(19):5787-98. doi: 10.1158/1078-0432.CCR-19-0070

61. Bray F, Ferlay J, Soerjomataram I, Siegel RL, Torre LA, Jemal A. Global cancer statistics 2018: GLOBOCAN estimates of incidence and mortality worldwide for 36 cancers in 185 countries. CA Cancer J Clin (2018) 68 (6):394-424. doi: 10.3322/caac.21492

62. Ferlay J, Colombet M, Soerjomataram I, Mathers C, Parkin DM, Pineros M, et al. Estimating the global cancer incidence and mortality in 2018: GLOBOCAN sources and methods. Int J Cancer (2019) 144(8):1941-53. doi: 10.1002/ijc.31937

63. Molina JR, Yang P, Cassivi SD, Schild SE, Adjei AA. Non-small cell lung cancer: epidemiology, risk factors, treatment, and survivorship. Mayo Clin Proc (2008) 83(5):584-94. doi: 10.4065/83.5.584

64. Topalian SL, Hodi FS, Brahmer JR, Gettinger SN, Smith DC, McDermott DF, et al. Five-Year Survival and Correlates Among Patients With Advanced Melanoma, Renal Cell Carcinoma, or Non-Small Cell Lung Cancer Treated With Nivolumab. JAMA Oncol (2019) 5(10):1411-20. doi: 10.1001/ jamaoncol.2019.2187

65. Garon EB, Hellmann MD, Rizvi NA, Carcereny E, Leighl NB, Ahn MJ, et al. Five-Year Overall Survival for Patients With Advanced NonSmall-Cell Lung Cancer Treated With Pembrolizumab: Results From the Phase I KEYNOTE001 Study. J Clin Oncol (2019) 37(28):2518-27. doi: 10.1200/JCO.19.00934

66. Brahmer JR, Johnson ML, Cobo M, Viteri S, Sarto JC, Sukari A, et al. JNJ64041757 (JNJ-757), a Live, Attenuated, Double-Deleted Listeria monocytogenes-Based Immunotherapy, in Patients With Non-Small Cell 
Lung Cancer: Results From 2 Phase 1 Studies. JTO Clin Res Rep (2020) 2:100103. doi: $10.1016 /$ j.jtocrr.2020.100103

67. Jafari F, Javdansirat S, Sanaie S, Naseri A, Shamekh A, Rostamzadeh D, et al. Osteosarcoma: A comprehensive review of management and treatment strategies. Ann Diagn Pathol (2020) 49:151654. doi: 10.1016/ j.anndiagpath.2020.151654

68. Link MP, Goorin AM, Miser AW, Green AA, Pratt CB, Belasco JB, et al. The effect of adjuvant chemotherapy on relapse-free survival in patients with osteosarcoma of the extremity. N Engl J Med (1986) 314(25):1600-6. doi: 10.1056/NEJM198606193142502

69. Dahlin DC, Coventry MB. Osteogenic sarcoma. A study of six hundred cases. J Bone Joint Surg Am (1967) 49(1):101-10. doi: 10.2106/00004623196749010-00008

70. Onda M, Matsuda S, Higaki S, Iijima T, Fukushima J, Yokokura A, et al. ErbB-2 expression is correlated with poor prognosis for patients with osteosarcoma. Cancer (1996) 77(1):71-8. doi: 10.1002/(SICI)1097-0142 (19960101)77:1<71::AID-CNCR13>3.0.CO;2-5

71. Simpson S, Dunning MD, de Brot S, Grau-Roma L, Mongan NP, Rutland CS. Comparative review of human and canine osteosarcoma: morphology, epidemiology, prognosis, treatment and genetics. Acta Vet Scand (2017) 59 (1):71. doi: 10.1186/s13028-017-0341-9

72. Flint AF, U'Ren L, Legare ME, Withrow SJ, Dernell W, Hanneman WH. Overexpression of the erbB-2 proto-oncogene in canine osteosarcoma cell lines and tumors. Vet Pathol (2004) 41(3):291-6. doi: 10.1354/vp.41-3-291

73. Shahabi V, Seavey MM, Maciag PC, Rivera S, Wallecha A. Development of a live and highly attenuated Listeria monocytogenes-based vaccine for the treatment of Her2/neu-overexpressing cancers in human. Cancer Gene Ther (2011) 18(1):53-62. doi: 10.1038/cgt.2010.48

74. Mason NJ, Gnanandarajah JS, Engiles JB, Gray F, Laughlin D, GaurnierHausser A, et al. Immunotherapy with a HER2-Targeting Listeria Induces HER2-Specific Immunity and Demonstrates Potential Therapeutic Effects in a Phase I Trial in Canine Osteosarcoma. Clin Cancer Res (2016) 22 (17):4380-90. doi: 10.1158/1078-0432.CCR-16-0088

75. Musser ML, Berger EP, Tripp CD, Clifford CA, Bergman PJ, Johannes CM. Safety evaluation of the canine osteosarcoma vaccine, live Listeria vector. Vet Comp Oncol (2020) 19(1):92-8. doi: 10.1111/vco.12642

76. Musser ML, Berger EP, Parsons C, Kathariou S, Johannes CM. Vaccine strain Listeria monocytogenes abscess in a dog: a case report. BMC Vet Res (2019) 15(1):467. doi: 10.1186/s12917-019-2216-y

77. Sinha S, Kuo C-Y, Ho JK, White PJ, Jazayeri JA, Pouton CW. A suicidal strain of Listeria monocytogenes is effective as a DNA vaccine delivery system for oral administration. Vaccine (2017) 35(38):5115-22. doi: 10.1016/ j.vaccine.2017.08.014

78. Hanson WG, Benanti EL, Lemmens EE, Liu W, Skoble J, Leong ML, et al. A Potent and Effective Suicidal Listeria Vaccine Platform. Infect Immun (2019) 87(8):e00144-19. doi: 10.1128/IAI.00144-19

79. Souders NC, Verch T, Paterson Y. In vivo bactofection: listeria can function as a DNA-cancer vaccine. DNA Cell Biol (2006) 25(3):142-51. doi: 10.1089/ dna.2006.25.142

80. Angelakopoulos H, Loock K, Sisul DM, Jensen ER, Miller JF, Hohmann EL. Safety and shedding of an attenuated strain of Listeria monocytogenes with a deletion of act $\mathrm{A} / \mathrm{plcB}$ in adult volunteers: a dose escalation study of oral inoculation. Infect Immun (2002) 70(7):3592-601. doi: 10.1128/ IAI.70.7.3592-3601.2002

81. Starks H, Bruhn KW, Shen H, Barry RA, Dubensky TW, Brockstedt D, et al. Listeria monocytogenes as a vaccine vector: virulence attenuation or existing antivector immunity does not diminish therapeutic efficacy. I Immunol (2004) 173(1):420-7. doi: 10.4049/jimmunol.173.1.420

82. Olino K, Wada S, Edil BH, Pan X, Meckel K, Weber W, et al. Tumorassociated antigen expressing Listeria monocytogenes induces effective primary and memory T-cell responses against hepatic colorectal cancer metastases. Ann Surg Oncol (2012) 19(Suppl 3):S597-607. doi: 10.1245/ s10434-011-2037-0

83. Golub SH, O'Connell TX, Morton DL. Correlation of in vivo and in vitro assays of immunocompetence in cancer patients. Cancer Res (1974) 34 (8):1833-7.

84. Dietrich G, Bubert A, Gentschev I, Sokolovic Z, Simm A, Catic A, et al. Delivery of antigen-encoding plasmid DNA into the cytosol of macrophages by attenuated suicide Listeria monocytogenes. Nat Biotechnol (1998) 16 (2):181-5. doi: 10.1038/nbt0298-181

85. Sacco JJ, Evans M, Harrington KJ, Man S, Powell N, Shaw RJ, et al. Systemic listeriosis following vaccination with the attenuated Listeria monocytogenes therapeutic vaccine, ADXS11-001. Hum Vaccin Immunother (2016) 12 (4):1085-6. doi: 10.1080/21645515.2016.1154248

86. Lim JY, Brockstedt DG, Lord EM, Gerber SA. Radiation therapy combined with Listeria monocytogenes-based cancer vaccine synergize to enhance tumor control in the B16 melanoma model. Oncoimmunology (2014) 3: e29028. doi: 10.4161/onci.29028

87. Burdett S, Rydzewska L, Tierney J, Fisher D, Parmar MK, Arriagada R, et al. Postoperative radiotherapy for non-small cell lung cancer. Cochrane Database Syst Rev (2016) 10:CD002142. doi: 10.1002/14651858.CD002142.pub4

88. Marmagkiolis K, Finch W, Tsitlakidou D, Josephs T, Iliescu C, Best JF, et al. Radiation Toxicity to the Cardiovascular System. Curr Oncol Rep (2016) 18 (3):15. doi: 10.1007/s11912-016-0502-4

89. Speer TW. Targeted Radionuclide Therapy. Philadelphia: Wolters Kluwer Health/Lippincott Williams \& Wilkins (2012).

90. Terán-Navarro H, Calderon-Gonzalez R, Salcines-Cuevas D, García I, Marradi M, Freire J, et al. Pre-clinical development of Listeria-based nanovaccines as immunotherapies for solid tumours: insights from melanoma. Oncoimmunology (2018) 8(2):e1541534-e. doi: 10.1080/ 2162402X.2018.1541534

91. Mandal M, Lee K-D. Listeriolysin O-liposome-mediated cytosolic delivery of macromolecule antigen in vivo: enhancement of antigen-specific cytotoxic $\mathrm{T}$ lymphocyte frequency, activity, and tumor protection. Biochim Biophys Acta (BBA) - Biomembranes (2002) 1563(1):7-17. doi: 10.1016/S0005-2736(02) 00368-1

92. Matsumura Y, Maeda H. A new concept for macromolecular therapeutics in cancer chemotherapy: mechanism of tumoritropic accumulation of proteins and the antitumor agent smancs. Cancer Res (1986) 46(12 Pt 1):6387-92.

93. Jain S, Hirst DG, O'Sullivan JM. Gold nanoparticles as novel agents for cancer therapy. Br J Radiol (2012) 85(1010):101-13. doi: 10.1259/bjr/ 59448833

94. La-Beck NM, Liu X, Wood LM. Harnessing Liposome Interactions With the Immune System for the Next Breakthrough in Cancer Drug Delivery. Front Pharmacol (2019) 10:220. doi: 10.3389/fphar.2019.00220

95. Yu YA, Shabahang S, Timiryasova TM, Zhang Q, Beltz R, Gentschev I, et al. Visualization of tumors and metastases in live animals with bacteria and vaccinia virus encoding light-emitting proteins. Nat Biotechnol (2004) 22 (3):313-20. doi: 10.1038/nbt937

96. Pilgrim S, Stritzker J, Schoen C, Kolb-Maurer A, Geginat G, Loessner MJ, et al. Bactofection of mammalian cells by Listeria monocytogenes: improvement and mechanism of DNA delivery. Gene Ther (2003) 10 (24):2036-45. doi: 10.4161/bbug.1.6.13146

97. Pálffy R, Gardlík R, Hodosy J, Behuliak M, Reško P, Radvánský J, et al. Bacteria in gene therapy: bactofection versus alternative gene therapy. Gene Ther (2006) 13(2):101-5. doi: 10.1038/sj.gt.3302635

98. Krusch S, Domann E, Frings M, Zelmer A, Diener M, Chakraborty T, et al. Listeria monocytogenes mediated CFTR transgene transfer to mammalian cells. J Gene Med (2002) 4(6):655-67. doi: 10.1002/jgm.313

99. Schoen C, Kolb-Mäurer A, Geginat G, Löffler D, Bergmann B, Stritzker J, et al. Bacterial delivery of functional messenger RNA to mammalian cells. Cell Microbiol (2005) 7(5):709-24. doi: 10.1111/j.1462-5822.2005.00507.x

100. Tangney M, van Pijkeren JP, Gahan CGM. The use of Listeria monocytogenes as a DNA delivery vector for cancer gene therapy. Bioeng Bugs (2010) 1 (4):284-7. doi: 10.4161/bbug.1.4.11725

101. van Pijkeren JP, Morrissey D, Monk IR, Cronin M, Cronin M, Rajendran S, et al. A novel Listeria monocytogenes-based DNA delivery system for cancer gene therapy. Hum Gene Ther (2010) 21(4):405-16. doi: 10.1089/ hum.2009.022

102. Loeffler DIM, Schoen CU, Goebel W, Pilgrim S. Comparison of Different Live Vaccine Strategies In Vivo for Delivery of Protein Antigen or AntigenEncoding DNA and mRNA by Virulence-Attenuated Listeria monocytogenes. Infect Immun (2006) 74(7):3946. doi: 10.1128/IAI.00112-06

103. Dagogo-Jack I, Shaw AT. Tumour heterogeneity and resistance to cancer therapies. Nat Rev Clin Oncol (2018) 15(2):81-94. doi: 10.1038/ nrclinonc.2017.166 
104. Zhang J, Adrian FJ, Jahnke W, Cowan-Jacob SW, Li AG, Iacob RE, et al. Targeting Bcr-Abl by combining allosteric with ATP-binding-site inhibitors. Nature (2010) 463(7280):501-6. doi: 10.1038/nature08675

105. Gong J, Chehrazi-Raffle A, Reddi S, Salgia R. Development of PD-1 and PDL1 inhibitors as a form of cancer immunotherapy: a comprehensive review of registration trials and future considerations. J Immunother Cancer (2018) 6 (1):8. doi: 10.1186/s40425-018-0316-Z

106. Wherry EJ. T cell exhaustion. Nat Immunol (2011) 12(6):492-9. doi: 10.1038/ni.2035

107. Li K, Tian H. Development of small-molecule immune checkpoint inhibitors of PD-1/PD-L1 as a new therapeutic strategy for tumour immunotherapy. J Drug Targeting (2019) 27(3):244-56. doi: 10.1080/1061186X.2018.1440400

108. Xu G, Feng D, Yao Y, Li P, Sun H, Yang H, et al. Listeria-based hepatocellular carcinoma vaccine facilitates anti-PD-1 therapy by regulating macrophage polarization. Oncogene (2020) 39(7):1429-44. doi: 10.1038/s41388-019$1072-3$

109. Kim VM, Blair AB, Lauer P, Foley K, Che X, Soares K, et al. Anti-pancreatic tumor efficacy of a Listeria-based, Annexin A2-targeting immunotherapy in combination with anti-PD-1 antibodies. J Immunother Cancer (2019) 7 (1):132. doi: 10.1186/s40425-019-0601-5

110. Mkrtichyan M, Chong N, Abu Eid R, Wallecha A, Singh R, Rothman J, et al. Anti-PD-1 antibody significantly increases therapeutic efficacy of Listeria monocytogenes (Lm)-LLO immunotherapy. J Immunother Cancer (2013) 1:15. doi: 10.1186/2051-1426-1-15

111. Gilley RP, Dube PH. Checkpoint blockade inhibitors enhances the effectiveness of a Listeria monocytogenes-based melanoma vaccine. Oncotarget (2020) 11(7):740-54. doi: 10.18632/oncotarget.27490

112. Kim CG, Kim C, Yoon SE, Kim KH, Choi SJ, Kang B, et al. Hyperprogressive disease during PD-1 blockade in patients with advanced hepatocellular carcinoma. J Hepatol (2021) 74(2):350-9. doi: 10.1016/j.jhep.2020.08.010

113. Finn RS, Ryoo BY, Merle P, Kudo M, Bouattour M, Lim HY, et al. Pembrolizumab As Second-Line Therapy in Patients With Advanced Hepatocellular Carcinoma in KEYNOTE-240: A Randomized, DoubleBlind, Phase III Trial. J Clin Oncol (2020) 38(3):193-202. doi: 10.1200/ JCO.19.01307

114. Rosenberg SA, Restifo NP. Adoptive cell transfer as personalized immunotherapy for human cancer. Science (2015) 348(6230):62-8. doi: 10.1126/science.aaa 4967

115. D'Ippolito E, Schober K, Nauerth M, Busch DH. T cell engineering for adoptive $\mathrm{T}$ cell therapy: safety and receptor avidity. Cancer Immunol Immunother (2019) 68(10):1701-12. doi: 10.1007/s00262-019-02395-9

116. Labani-Motlagh A, Ashja-Mahdavi M, Loskog A. The Tumor Microenvironment: A Milieu Hindering and Obstructing Antitumor Immune Responses. Front Immunol (2020) 11:940. doi: 10.3389/ fimmu.2020.00940

117. Jahangir A, Chandra D, Quispe-Tintaya W, Singh M, Selvanesan BC, Gravekamp C. Immunotherapy with Listeria reduces metastatic breast cancer in young and old mice through different mechanisms. Oncoimmunology (2017) 6(9):e1342025. doi: 10.1080/2162402X. 2017.1342025

118. Sotillo E, Barrett DM, Black KL, Bagashev A, Oldridge D, Wu G, et al. Convergence of Acquired Mutations and Alternative Splicing of CD19 Enables Resistance to CART-19 Immunotherapy. Cancer Discov (2015) 5 (12):1282-95. doi: 10.1158/2159-8290.CD-15-1020

119. Walsh SR, Simovic B, Chen L, Bastin D, Nguyen A, Stephenson K, et al. Endogenous $\mathrm{T}$ cells prevent tumor immune escape following adoptive $\mathrm{T}$ cell therapy. J Clin Invest (2019) 129(12):5400-10. doi: 10.1172/JCI126199

120. Lu S. Heterologous prime-boost vaccination. Curr Opin Immunol (2009) 21 (3):346-51. doi: 10.1016/j.coi.2009.05.016

121. Liu J, Ewald BA, Lynch DM, Denholtz M, Abbink P, Lemckert AA, et al. Magnitude and phenotype of cellular immune responses elicited by recombinant adenovirus vectors and heterologous prime-boost regimens in rhesus monkeys. J Virol (2008) 82(10):4844-52. doi: 10.1128/JVI.02616-07

122. Johnson LE, Brockstedt D, Leong M, Lauer P, Theisen E, Sauer JD, et al. Heterologous vaccination targeting prostatic acid phosphatase (PAP) using DNA and Listeria vaccines elicits superior anti-tumor immunity dependent on CD4+T cells elicited by DNA priming. Oncoimmunology (2018) 7(8):210. doi: $10.1080 / 2162402 X .2018 .1456603$
123. Aitken AS, Roy DG, Martin NT, Sad S, Bell JC, Bourgeois-Daigneault MC. Brief Communication; A Heterologous Oncolytic Bacteria-Virus PrimeBoost Approach for Anticancer Vaccination in Mice. J Immunother (2018) 41(3):125-9. doi: 10.1097/CJI.0000000000000208

124. Ishizaki H, Song GY, Srivastava T, Carroll KD, Shahabi V, Manuel ER, et al. Heterologous prime/boost immunization with p53-based vaccines combined with toll-like receptor stimulation enhances tumor regression. J Immunother (2010) 33(6):609-17. doi: 10.1097/CJI.0b013e3181e032c6

125. Zebertavage L, Bambina S, Shugart J, Alice A, Zens KD, Lauer P, et al. A microbial-based cancer vaccine for induction of EGFRvIII-specific CD8+ T cells and anti-tumor immunity. PloS One (2019) 14(1):e0209153. doi: 10.1371/journal.pone.0209153

126. Connell PP, Hellman S. Advances in radiotherapy and implications for the next century: a historical perspective. Cancer Res (2009) 69(2):383-92. doi: 10.1158/0008-5472.CAN-07-6871

127. Kumari S, Mukherjee S, Sinha D, Abdisalaam S, Krishnan S, Asaithamby A. Immunomodulatory Effects of Radiotherapy. Int J Mol Sci (2020) 21(21):11-7. doi: $10.3390 /$ ijms 21218151

128. Garnett-Benson C, Hodge JW, Gameiro SR. Combination regimens of radiation therapy and therapeutic cancer vaccines: mechanisms and opportunities. Semin Radiat Oncol (2015) 25(1):46-53. doi: 10.1016/ j.semradonc.2014.07.002

129. Barker HE, Paget JT, Khan AA, Harrington KJ. The tumour microenvironment after radiotherapy: mechanisms of resistance and recurrence. Nat Rev Cancer (2015) 15(7):409-25. doi: 10.1038/nrc3958

130. Hannan R, Zhang H, Wallecha A, Singh R, Liu L, Cohen P, et al. Combined immunotherapy with Listeria monocytogenes-based PSA vaccine and radiation therapy leads to a therapeutic response in a murine model of prostate cancer. Cancer Immunol Immunother (2012) 61(12):2227-38. doi: 10.1007/s00262-012-1257-x

131. Merle NS, Noe R, Halbwachs-Mecarelli L, Fremeaux-Bacchi V, Roumenina LT. Complement System Part II: Role in Immunity. Front Immunol (2015) 6:257. doi: 10.3389/fimmu.2015.00257

132. Markiewski MM, DeAngelis RA, Benencia F, Ricklin-Lichtsteiner SK, Koutoulaki A, Gerard C, et al. Modulation of the antitumor immune response by complement. Nat Immunol (2008) 9(11):1225-35. doi: 10.1038/ni.1655

133. Wang Y, Sun SN, Liu Q, Yu YY, Guo J, Wang K, et al. Autocrine Complement Inhibits IL10-Dependent T-cell-Mediated Antitumor Immunity to Promote Tumor Progression. Cancer Discov (2016) 6 (9):1022-35. doi: 10.1158/2159-8290.CD-15-1412

134. Reese B, Silwal A, Daugherity E, Daugherity M, Arabi M, Daly P, et al. Complement as Prognostic Biomarker and Potential Therapeutic Target in Renal Cell Carcinoma. J Immunol (2020) 205(11):3218-29. doi: 10.4049/ jimmunol.2000511

135. Ghouse SM, Vadrevu SK, Manne S, Reese B, Patel J, Patel B, et al. Therapeutic Targeting of Vasculature in the Premetastatic and Metastatic Niches Reduces Lung Metastasis. J Immunol (2020) 204(4):990-1000. doi: 10.4049/jimmunol.1901208

136. Nowak AK, Robinson BWS, Lake RA. Synergy between Chemotherapy and Immunotherapy in the Treatment of Established Murine Solid Tumors. Cancer Res (2003) 63(15):4490.

137. Nowak AK, Lake RA, Marzo AL, Scott B, Heath WR, Collins EJ, et al. Induction of Tumor Cell Apoptosis In Vivo Increases Tumor Antigen CrossPresentation, Cross-Priming Rather than Cross-Tolerizing Host TumorSpecific CD8 T Cells. J Immunol (2003) 170(10):4905. doi: 10.4049/ jimmunol.170.10.4905

138. Le DT, Jaffee EM. Regulatory T-cell modulation using cyclophosphamide in vaccine approaches: a current perspective. Cancer Res (2012) 72(14):3439_ 44. doi: 10.1158/0008-5472.CAN-11-3912

139. Yoshimura K, Laird LS, Chia CY, Meckel KF, Slansky JE, Thompson JM, et al. Live attenuated Listeria monocytogenes effectively treats hepatic colorectal cancer metastases and is strongly enhanced by depletion of regulatory T cells. Cancer Res (2007) 67(20):10058-66. doi: 10.1158/00085472.CAN-07-0573

140. Keenan BP, Saenger Y, Kafrouni MI, Leubner A, Lauer P, Maitra A, et al. A Listeria vaccine and depletion of T-regulatory cells activate immunity against early stage pancreatic intraepithelial neoplasms and prolong 
survival of mice. Gastroenterology (2014) 146(7):1784-94 e6. doi: 10.1053/ j.gastro.2014.02.055

141. Hingorani SR, Wang L, Multani AS, Combs C, Deramaudt TB, Hruban RH, et al. Trp53R172H and KrasG12D cooperate to promote chromosomal instability and widely metastatic pancreatic ductal adenocarcinoma in mice. Cancer Cell (2005) 7(5):469-83. doi: 10.1016/j.ccr.2005.04.023

142. Beatty GL, Gladney WL. Immune escape mechanisms as a guide for cancer immunotherapy. Clin Cancer Res (2015) 21(4):687-92. doi: 10.1158/10780432.CCR-14-1860

143. Singh R, Paterson Y. Immunoediting sculpts tumor epitopes during immunotherapy. Cancer Res (2007) 67(5):1887-92. doi: 10.1158/00085472.CAN-06-3960

144. Kim SH, Castro F, Paterson Y, Gravekamp C. High efficacy of a Listeriabased vaccine against metastatic breast cancer reveals a dual mode of action. Cancer Res (2009) 69(14):5860-6. doi: 10.1158/0008-5472.CAN-08-4855
Conflict of Interest: YP has a financial interest in Advaxis, Inc., a vaccine and therapeutic company that has licensed or has an option to license all patents from the University of Pennsylvania that concern the use of Listeria monocytogenes or listerial products as vaccines.

The remaining authors declare that the research was conducted in the absence of any commercial or financial relationships that could be construed as a potential conflict of interest.

Copyright (๑) 2021 Oladejo, Paterson and Wood. This is an open-access article distributed under the terms of the Creative Commons Attribution License (CC BY). The use, distribution or reproduction in other forums is permitted, provided the original author(s) and the copyright owner(s) are credited and that the original publication in this journal is cited, in accordance with accepted academic practice. No use, distribution or reproduction is permitted which does not comply with these terms. 